\title{
Bayesian Exponential Random Graph Models with Nodal Random Effects
}

\author{
Stephanie Thiemichen ${ }^{a}$, Nial Friel ${ }^{b}$, Alberto Caimo ${ }^{c}$, Göran Kauermann ${ }^{a}$. \\ ${ }^{a}$ Institut für Statistik, Ludwigs-Maximilians-Universität München, Germany. \\ ${ }^{b}$ School of Mathematical Sciences and Insight: The National Centre for Data Analytics, \\ University College Dublin, Ireland. \\ ${ }^{c}$ School of Mathematical Sciences, Dublin Institute of Technology, Ireland.
}

December 21, 2015

\begin{abstract}
We extend the well-known and widely used Exponential Random Graph Model (ERGM) by including nodal random effects to compensate for heterogeneity in the nodes of a network. The Bayesian framework for ERGMs proposed by Caimo and Friel (2011) yields the basis of our modelling algorithm. A central question in network models is the question of model selection and following the Bayesian paradigm we focus on estimating Bayes factors. To do so we develop an approximate but feasible calculation of the Bayes factor which allows one to pursue model selection. Three data examples and a small simulation study illustrate our mixed model approach and the corresponding model selection.
\end{abstract}

Keywords: Exponential random graph models; Bayesian inference; Random effects; Network analysis

Corresponding author:

Stephanie Thiemichen

Institut für Statistik, Ludwigs-Maximilians-Universität München

Ludwigstr. 33, 80539 München, Germany

Telephone number: +49 - (0)89 - 2180 2232, Fax number: +49 - (0)89 - 21805040

stephanie.thiemichen@stat.uni-muenchen.de 
NOTE: Changes to original version are set in bold.

\section{Introduction}

The analysis of network data is an emerging field in statistics which is challenging both model-wise and computationally. Recently Goldenberg et al. (2010), Hunter et al. (2012), Fienberg (2012), and Salter-Townshend et al. (2012), respectively, published comprehensive survey articles discussing statistical approaches, challenges and developments in network data analysis. We also refer to the monograph of Kolaczyk (2009) for a comprehensive introduction to the field.

In this paper we consider networks represented as a $n \times n$ dimensional adjacency matrix $\boldsymbol{Y}$, where the element $Y_{i j}=1$, if an edge exists between vertex $i$ and vertex $j$, and $Y_{i j}=0$ otherwise, with $i, j \in\{1, \ldots, n\}$ and $i \neq j$, that is there is no connection from a vertex to itself. With $n$ we denote the number of vertices in the network and for simplicity we assume undirected edges, that is $Y_{i j}=Y_{j i}$. Therefore, the matrix $\boldsymbol{Y}$ is symmetric and for simplicity it is sufficient to consider the upper triangle of $\boldsymbol{Y}$ only, that is $Y_{i j}, j>i$. Our approach equally applies to non-symmetric adjacency matrices corresponding to directed graphs. A concrete realisation of $\boldsymbol{Y}$ is denoted with $\boldsymbol{y}$.

With respect to the available statistical models for modelling cross-sectional network data one may roughly distinguish between two strands, (a) models which explain the existence of an edge purely with external nodal covariates or random effects and (b) models where the existence of an edge also depends on the local network structure. The first strand of models is phrased as $p_{1}$ and $p_{2}$ models tracing back to Holland and Leinhardt (1981). Specifically, in the $p_{1}$ model we set

$$
\operatorname{logit}\left[\mathbb{P}\left(Y_{i j}=1\right)\right]=\log \left\{\frac{\mathbb{P}\left(Y_{i j}=1\right)}{1-\mathbb{P}\left(Y_{i j}=1\right)}\right\}=\alpha_{i}+\alpha_{j}+\boldsymbol{z}_{i j}^{t} \boldsymbol{\beta}
$$

where $\boldsymbol{z}_{i j}$ denotes a set of covariates relating to the vertices $i$ and $j$ and $\alpha_{i}$ and $\alpha_{j}$ are nodal effects, here assuming undirected edges. Since the number of parameters increases with increasing network size $n$, van Duijn et al. (2004) proposed to replace the $\alpha$ parameters in (1) by random effects, see also Zijlstra et al. (2006). This yields the $p_{2}$ model

$$
\begin{gathered}
\operatorname{logit}\left[\mathbb{P}\left(Y_{i j}=1 \mid \phi\right)\right]=\phi_{i}+\phi_{j}+\boldsymbol{z}_{i j}^{t} \beta, \\
\boldsymbol{\phi}=\left(\phi_{1}, \ldots, \phi_{n}\right)^{t} \sim N\left(0, \sigma_{\phi}^{2} I_{n}\right)
\end{gathered}
$$

with $I_{n}$ as $n$ dimensional unit matrix. A general principle with this approach is that vertices (or actors in the network, respectively) are not considered as homogeneous but heterogeneous, though their heterogeneity is not observable but latent and expressed in the node specific random effects $\phi_{i}, i=1, \ldots, n$.

Both, the $p_{1}$ and the $p_{2}$ model lie within the classical generalized linear (mixed) model framework which allows estimation using standard statistical software. The $p_{2}$ models also allow for Bayesian estimation approaches, see for example Gill and Swartz (2004). 
The second strand in statistical network modelling is based on the so called Exponential Random Graph Model (ERGM) proposed by Frank and Strauss (1986). Here we model directly the network using the likelihood function

$$
\mathbb{P}(\boldsymbol{Y}=\boldsymbol{y} \mid \boldsymbol{\theta})=f(\boldsymbol{y} \mid \boldsymbol{\theta})=\frac{q_{\boldsymbol{\theta}}(\boldsymbol{y})}{\kappa(\boldsymbol{\theta})}=\frac{\exp \left\{\boldsymbol{\theta}^{t} s(\boldsymbol{y})\right\}}{\kappa(\boldsymbol{\theta})}
$$

where $\boldsymbol{\theta}=\left(\theta_{0}, \ldots, \theta_{p}\right)^{t}$ is the vector of model parameters and $s(\boldsymbol{y})=\left(s_{0}(\boldsymbol{y}), \ldots, s_{p}(\boldsymbol{y})\right)^{t}$ is a vector of sufficient network statistics like the number of edges or two-stars in a network, see for example Snijders et al. (2006). In equation (3) the term $\kappa(\boldsymbol{\theta})$ denotes the normalizing constant, that is

$$
\kappa(\boldsymbol{\theta})=\sum_{\boldsymbol{y} \in \mathcal{Y}} \exp \left\{\boldsymbol{\theta}^{t} s(\boldsymbol{y})\right\}
$$

and is accordingly the sum over $2^{\left(\begin{array}{l}n \\ 2\end{array}\right)}$ potential undirected graphs and therefore numerically intractable, except for very small graphs. Early fitting approaches are based on the pseudolikelihood idea proposed by Strauss and Ikeda (1990). More advanced are MCMC based routines proposed by Hunter and Handcock (2006) based on the work of Geyer and Thompson (1992). A fully Bayesian approach to estimate ERGMs has been developed by Caimo and Friel (2011).

Model (3) allows for a conditional interpretation by focusing on the occurrence of a single edge between two nodes. To be specific we obtain

$$
\operatorname{logit}\left[\mathbb{P}\left(Y_{i j}=1 \mid Y_{k l},(k, l) \neq(i, j) ; \boldsymbol{\theta}\right)\right]=\boldsymbol{\theta}^{t} s_{i j}(\boldsymbol{y}),
$$

where $s_{i j}(\boldsymbol{y})$ denotes the vector of so called change statistics

$$
s_{i j}(\boldsymbol{y})=s\left(y_{i j}=1, y_{k l},(k, l) \neq(i, j)\right)-s\left(y_{i j}=0, y_{k l},(k, l) \neq(i, j)\right) .
$$

We refer to Robins et al. (2007a), Robins et al. (2007b), and the rather recent work of Lusher et al. (2013) for a deeper discussion of Exponential Random Graph Models.

Contrasting equation (4) with the $p_{1}$ and $p_{2}$ model given in equations (1) and (2) it becomes obvious that the ERGM in contrast to the $p_{1}$ and $p_{2}$ models take the network structure into account while considering the nodes to be homogeneous. When modelling network data this means that all possible heterogeneity in the network nodes (that is the actors in the network) is included as covariates in the model and influence the (global) structure of the network. Since homogeneity of the nodes have led from $p_{1}$ to $p_{2}$ models, we want to pursue the same modelling exercise by allowing for latent node specific heterogeneity in Exponential Random Graph Models. To do so, we combine the $p_{2}$ model (2) with the ERGM (4) towards

$$
\operatorname{logit}\left[\mathbb{P}\left(Y_{i j}=1 \mid Y_{k l},(k, l) \neq(i, j) ; \boldsymbol{\theta}, \phi_{i}, \phi_{j}\right)\right]=\boldsymbol{\theta}^{t} s_{i j}(\boldsymbol{y})+\phi_{i}+\phi_{j}
$$

with $\phi=\left(\phi_{1}, \ldots, \phi_{n}\right)^{t}$ and $\phi_{i} \stackrel{\text { i.i.j. }}{\sim} N\left(\mu_{\phi}, \sigma_{\phi}^{2}\right), i=1, \ldots, n$. The parameter $\mu_{\phi}$ captures the average propensity in the network for forming a tie. Therefore $\theta_{0}$, which is usually the 
parameter associated with the edges statistic, is excluded from $\boldsymbol{\theta}$, i.e. $\boldsymbol{\theta}=\left(\theta_{1}, \ldots, \theta_{p}\right)^{t}$ here. In terms of the likelihood function for the whole network we obtain from (5)

$$
\mathbb{P}(\boldsymbol{Y}=\boldsymbol{y} \mid \boldsymbol{\theta}, \boldsymbol{\phi})=f(\boldsymbol{y} \mid \boldsymbol{\theta}, \boldsymbol{\phi})=\frac{q_{\boldsymbol{\theta}, \boldsymbol{\phi}}(\boldsymbol{y})}{\kappa(\boldsymbol{\theta}, \boldsymbol{\phi})}=\frac{\exp \left\{\boldsymbol{\theta}^{t} s(\boldsymbol{y})+\boldsymbol{\phi}^{t} t(\boldsymbol{y})\right\}}{\kappa(\boldsymbol{\theta}, \boldsymbol{\phi})}
$$

where $t(\boldsymbol{y})$ contains the degree statistics of the $n$ vertices, i.e. $t_{i}(\boldsymbol{y})=\sum_{j=1}^{n} y_{i j}$, for $i=$ $1, \ldots, n$. That is we fit an Exponential Random Graph Model with random, node specific effects accounting for heterogeneity. The model in equations (5) and (6) falls in the general class of Exponential-family Random Network Models proposed by Fellows and Handcock (2012) but unlike their model we treat the node specific effect as latent and we pursue a fully Bayesian estimation. We also refer to Krivitsky et al. (2009) who propose a model with actor specific random effects based on a latent cluster model. The authors also propose node specific random effects. We follow this line and give further interpretability of the effects. A central issue in model extensions is the question of model selection. We emphasize this point in the paper by comparing models with and without nodal effects using the Bayes factor as model selection criterion. However, calculation of the Bayes factor suffers from the above mentioned problem in Exponential Random Graph Models in that the normalization constant $\kappa(\cdot)$ is numerically infeasible. We therefore propose an approximate calculation of the Bayes factor and show in a simulation study its usability for model selection.

For estimation and model selection of model (6) we extend the fully Bayesian approach from Caimo and Friel (2011). The developed estimation routine is based on the numerical work of Caimo and Friel (2014) with their R (R Core Team, 2015) package Bergm (see http://cran.r-project.org/web/packages/Bergm). Our algorithms for model fitting and selection will be included in the Bergm package.

The paper is organized as follows. In Section 2 we derive a fully Bayesian formulation of the model. This is followed by a detailed description of the MCMC based estimation routine. Section 3 deals with the issue of model selection using Bayes factors. Three data examples and some simulation results are presented Section 4 . Finally Section 5 concludes with a discussion.

\section{Bayesian model formulation and Estimation}

Before proposing a fully Bayesian formulation for model (6) bear in mind that the normalizing constant $\kappa(\boldsymbol{\theta}, \boldsymbol{\phi})$ is numerically infeasible to calculate except for small networks so that numerically demanding simulation based fitting routines need to be employed. We follow a fully Bayesian approach by imposing a prior distribution on $\boldsymbol{\theta}=\left(\theta_{1}, \ldots, \theta_{p}\right)^{t}$. The posterior of interest for the Bayesian Exponential Random Graph 
Model with nodal random effects in (6) then becomes

$$
p\left(\boldsymbol{\theta}, \boldsymbol{\phi}, \mu_{\phi}, \sigma_{\phi}^{2} \mid \boldsymbol{y}\right)=\frac{f(\boldsymbol{y} \mid \boldsymbol{\theta}, \boldsymbol{\phi}) p(\boldsymbol{\theta}) p\left(\boldsymbol{\phi} \mid \mu_{\phi}, \sigma_{\phi}^{2}\right) p\left(\mu_{\phi}\right) p\left(\sigma_{\phi}^{2}\right)}{p(\boldsymbol{y})},
$$

where $p(\boldsymbol{\theta})$ is the prior distribution of $\boldsymbol{\theta}$ and $p\left(\phi \mid \mu_{\phi}, \sigma_{\phi}^{2}\right)$ the prior for the random nodal effects $\phi$. We assume the nodal effects to be independent and identically normally distributed, that is

$$
\phi_{i} \sim N\left(\mu_{\phi}, \sigma_{\phi}^{2}\right), \quad \text { for } i=1, \ldots, n
$$

and accordingly we use $\boldsymbol{\theta} \sim N\left(0, \rho^{2} I_{p}\right)$, with $I_{p}$ denoting the $p$-dimensional unity matrix and $\rho^{2}$ chosen such that the prior distribution is flat. For the hyper prior distribution $p\left(\mu_{\phi}\right)$ of the mean $\mu_{\phi}$ we assume a normal distribution centred at 0 , that is

$$
\mu_{\phi} \sim N\left(0, \tau^{2}\right)
$$

The hyper prior $p\left(\sigma_{\phi}^{2}\right)$ of the variance $\sigma_{\phi}^{2}$ is assumed to be an inverse gamma distribution, that is

$$
\sigma_{\phi}^{2} \sim I G(a, b)
$$

Finally, the parameters $\tau^{2}, a$ and $b$ are all constants and chosen in a way that results in flat hyper prior distributions. Figure 1 illustrates this Bayesian model formulation.

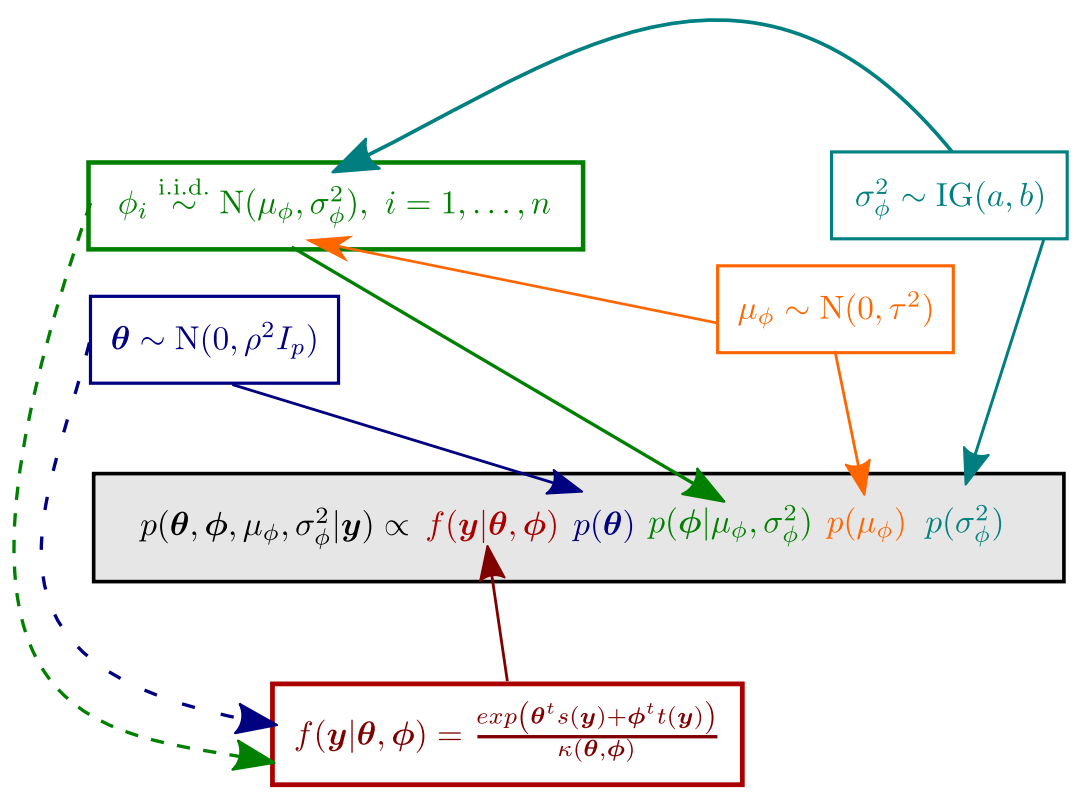

Figure 1 Overview of the Bayesian model formulation for the Exponential Random Graph Model with nodal random effects.

It is important to note, that the posterior distribution in (7) is so-called doublyintractable. This is because, firstly, it is not possible to evaluate the posterior density (7) due to $p(\boldsymbol{y})$, the marginal likelihood or evidence, being intractable. Secondly, it is 
also numerically infeasible to calculate the normalizing constant $\kappa(\boldsymbol{\theta}, \boldsymbol{\phi})$ in the likelihood $f(\boldsymbol{y} \mid \boldsymbol{\theta}, \boldsymbol{\phi})$ except for very small network graphs. Similar to the algorithm proposed by Caimo and Friel (2011) we use the so-called exchange algorithm from Murray et al. (2006) to draw samples from the posterior distribution of interest. Let therefore $\gamma=\left(\boldsymbol{\theta}^{t}, \boldsymbol{\phi}^{t}\right)^{t}$ denote the entire parameter vector of the ERGM. Instead of drawing directly from ( 7$)$, we sample from the augmented distribution

$$
\begin{aligned}
& p\left(\boldsymbol{\gamma}^{\prime}, \boldsymbol{y}^{\prime}, \boldsymbol{\gamma}, \mu_{\phi}, \sigma_{\phi}^{2} \mid \boldsymbol{y}\right) \propto \\
& \quad f(\boldsymbol{y} \mid \boldsymbol{\gamma}) p\left(\boldsymbol{\gamma} \mid \mu_{\phi}, \sigma_{\phi}^{2}\right) p\left(\mu_{\phi}\right) p\left(\sigma_{\phi}^{2}\right) h\left(\boldsymbol{\gamma}^{\prime} \mid \boldsymbol{\gamma}\right) f\left(\boldsymbol{y}^{\prime} \mid \boldsymbol{\gamma}^{\prime}\right),
\end{aligned}
$$

where $h(\cdot \mid \cdot)$ is a proposal function, to be specified later. This proposal provides $\boldsymbol{\gamma}^{\prime}=\left(\boldsymbol{\theta}^{\prime t}, \boldsymbol{\phi}^{\prime t}\right)^{t}$ as new candidate values for $\boldsymbol{\theta}$ and $\boldsymbol{\phi}$, respectively, and based on $\boldsymbol{\gamma}^{\prime}$ we can simulate $\boldsymbol{y}^{\prime}$ as an auxiliary network. The proposal is accepted with probability

$$
\alpha=\min \left(1, \frac{q_{\boldsymbol{\gamma}}\left(\boldsymbol{y}^{\prime}\right) p\left(\boldsymbol{\gamma}^{\prime}\right) h\left(\boldsymbol{\gamma} \mid \boldsymbol{\gamma}^{\prime}\right) q_{\boldsymbol{\gamma}^{\prime}}(\boldsymbol{y})}{q_{\boldsymbol{\gamma}}(\boldsymbol{y}) p(\boldsymbol{\gamma}) h\left(\boldsymbol{\gamma}^{\prime} \mid \boldsymbol{\gamma}\right) q_{\gamma^{\prime}}\left(\boldsymbol{y}^{\prime}\right)} \times \frac{\kappa(\boldsymbol{\gamma}) \kappa\left(\boldsymbol{\gamma}^{\prime}\right)}{\kappa(\boldsymbol{\gamma}) \kappa\left(\boldsymbol{\gamma}^{\prime}\right)}\right)
$$

where $p(\boldsymbol{\gamma})=p(\boldsymbol{\theta}) \cdot p\left(\boldsymbol{\phi} \mid \mu_{\phi}, \sigma_{\phi}^{2}\right)$. Note that in (9) the normalizing constants cancel out so that (9) is in principle easy to calculate. Though the algorithm is in this form a direct extension of the algorithm for Bayesian Exponential Random Graph Models of Caimo and Friel (2011) it is advisable to separate the proposals of $\boldsymbol{\theta}$ and $\boldsymbol{\phi}$ to achieve higher acceptance rates. This is described in the following algorithmic steps. In detail, our algorithm works as follows:

\section{Algorithm 1: Fit BERGM with nodal random effects}

Step 1: Gibbs update of $\left(\boldsymbol{\theta}^{\prime}, \boldsymbol{y}^{\prime}\right)$ :

(i) Draw $\boldsymbol{\theta}^{\prime} \sim h(\cdot \mid \boldsymbol{\theta})$.

(ii) Draw $\boldsymbol{y}^{\prime} \sim p\left(\cdot \mid \boldsymbol{\theta}^{\prime}, \boldsymbol{\phi}\right)$.

(iii) Propose to move from $\boldsymbol{\theta}$ to $\boldsymbol{\theta}^{\prime}$ with probability

$$
\alpha=\min \left(1, \frac{q_{\boldsymbol{\theta}, \boldsymbol{\phi}}\left(\boldsymbol{y}^{\prime}\right) p\left(\boldsymbol{\theta}^{\prime}\right) h\left(\boldsymbol{\theta} \mid \boldsymbol{\theta}^{\prime}\right) q_{\boldsymbol{\theta}^{\prime}, \boldsymbol{\phi}}(\boldsymbol{y})}{q_{\boldsymbol{\theta}, \phi}(\boldsymbol{y}) p(\boldsymbol{\theta}) h\left(\boldsymbol{\theta}^{\prime} \mid \boldsymbol{\theta}\right) q_{\boldsymbol{\theta}^{\prime}, \boldsymbol{\phi}}\left(\boldsymbol{y}^{\prime}\right)} \times \frac{\kappa(\boldsymbol{\theta}, \boldsymbol{\phi}) \kappa\left(\boldsymbol{\theta}^{\prime}, \boldsymbol{\phi}\right)}{\kappa(\boldsymbol{\theta}, \boldsymbol{\phi}) \kappa\left(\boldsymbol{\theta}^{\prime}, \boldsymbol{\phi}\right)}\right) .
$$

Step 2: Gibbs update of $\left(\boldsymbol{\phi}^{\prime}, \boldsymbol{y}^{\prime}\right)$ :

(i) Draw $\boldsymbol{\phi}^{\prime} \sim g(\cdot \mid \phi)$.

(ii) Draw $\boldsymbol{y}^{\prime} \sim p\left(\cdot \mid \boldsymbol{\theta}, \boldsymbol{\phi}^{\prime}\right)$.

(iii) Propose to move from $\phi$ to $\phi^{\prime}$ with probability

$$
\alpha=\min \left(1, \frac{q_{\boldsymbol{\theta}, \phi}\left(\boldsymbol{y}^{\prime}\right) p\left(\boldsymbol{\phi}^{\prime} \mid \mu_{\phi}, \sigma_{\phi}^{2}\right) g\left(\boldsymbol{\phi} \mid \boldsymbol{\phi}^{\prime}\right) q_{\boldsymbol{\theta}, \phi^{\prime}}(\boldsymbol{y})}{q_{\boldsymbol{\theta}, \boldsymbol{\phi}}(\boldsymbol{y}) p\left(\boldsymbol{\phi} \mid \mu_{\phi}, \sigma_{\phi}^{2}\right) g\left(\boldsymbol{\phi}^{\prime} \mid \boldsymbol{\phi}\right) q_{\boldsymbol{\theta}, \boldsymbol{\phi}^{\prime}}\left(\boldsymbol{y}^{\prime}\right)} \times \frac{\kappa(\boldsymbol{\theta}, \boldsymbol{\phi}) \kappa\left(\boldsymbol{\theta}, \boldsymbol{\phi}^{\prime}\right)}{\kappa(\boldsymbol{\theta}, \boldsymbol{\phi}) \kappa\left(\boldsymbol{\theta}, \boldsymbol{\phi}^{\prime}\right)}\right)
$$


Step 3: Metropolis-Hastings update of $\mu_{\phi}$ :

Draw proposal $\mu_{\phi}^{\prime}$ from $k\left(\cdot \mid \mu_{\phi}\right)$ and accept the proposed value with probability

$$
\alpha=\min \left(1, \frac{p\left(\phi \mid \mu_{\phi}, \sigma_{\phi}^{2}\right) p\left(\mu_{\phi}\right)}{p\left(\phi \mid \mu_{\phi}^{\prime}, \sigma_{\phi}^{2}\right) p\left(\mu_{\phi}^{\prime}\right)}\right) .
$$

Step 4: Metropolis-Hastings update of $\sigma_{\phi}^{2}$ :

Draw proposal $\sigma_{\phi}^{2 \prime}$ from $l\left(\cdot \mid \sigma_{\phi}^{2}\right)$ and accept the proposed value with probability

$$
\alpha=\min \left(1, \frac{p\left(\phi \mid \mu_{\phi}, \sigma_{\phi}^{2}\right) p\left(\sigma_{\phi}^{2}\right)}{p\left(\phi \mid \mu_{\phi}, \sigma_{\phi}^{2 \prime}\right) p\left(\sigma_{\phi}^{2^{\prime}}\right)}\right) .
$$

Start again with Step 1 until the maximum number of iterations is reached.

It is easy to see that there is no necessity to compute the normalizing constants $\kappa(\cdot)$, because they cancel out when calculating the acceptance probabilities in the first two steps of the algorithm. The current implementation of the algorithm uses single-site updates for the update of $\phi$, that is each $\phi_{i}, i=1, \ldots, n$ is updated in turn while all other values are kept constant. This leads to reasonable acceptance probabilities for the Markov chain.

The default choices for the proposal functions $h(\cdot \mid \cdot), g(\cdot \mid \cdot)$ and $k(\cdot \mid \cdot)$ are normal distributions centred at the current parameter value, for $l(\cdot \mid \cdot)$ we use a uniform distribution, which is symmetric around the current value of $\sigma_{\phi}^{2}$ and truncated at zero to avoid negative proposals for the variance parameter.

The draws of the auxiliary network $\boldsymbol{y}^{\prime}$ in the component of steps 1 and 3 are realised using the "tie no tie" sampler from the ergm package (Hunter et al., 2008), which is a simple Gibbs sampler. Although this auxiliary Gibbs sampler does not yield an exact draw $\boldsymbol{y}^{\prime}$, Everitt (2012) has shown, under some assumptions, that the resulting approximate exchange algorithm converges to the target distribution as the number of auxiliary draws tends to infinity. As a practical result he points out that for the number of auxiliary iterations it is often sufficient to use roughly the number of possible ties in the network.

\section{Model Selection}

\subsection{Bayesian Model Selection}

Model Selection is an important, often neglected issue in network data analysis. We put special emphasis on this task here and propose the Bayes factor suitable for model selection. One of the interesting questions in our model is, if we are able to distinguish the three following model generating processes: 
(1) Nodal random effects only, i.e. the $p_{2}$ model,

(2) Structural effects only, i.e. the standard ERGM, and

(3) ERGM in combination with nodal random effects.

This question results in the problem of model selection. The data examples in Section 4.1 illustrate this issue.

Classical Bayesian tools for model comparison such as the deviance information criterion (DIC) as suggested by Spiegelhalter et al. (2002) are not directly available, again due to the intractability of the normalizing constant of the likelihood in model equation (6).

Computing Bayes factors for model choice using reversible jump Markov Chain Monte Carlo for Bayesian Exponential Random Graph Models as done by Caimo and Friel (2013) is not an option for our model. This approach would be possible in general, but very time consuming from a computational point of view.

In the following subsections we present two approaches for model selection based on Bayes factors, one for nested models, and a more general approach for non-nested models.

\subsection{Bayes Factor for Nested Models}

We suggest the following strategy for deciding whether to include nodal random effects into the model or not. The goal is to calculate a Bayes factor for two competing models (Kass and Raftery, 1995). First we fit the two Exponential Random Graph Models, one with edges and non-random effects only, notated as model $m_{1}$ with coefficients $\boldsymbol{\theta}^{\prime}=\left(\theta_{0}^{\prime}, \ldots, \theta_{p}^{\prime}\right)^{t}$, and the second one with nodal random effects instead of the edges term, labelled as model $m_{2}$ with coefficients $\boldsymbol{\theta}=\left(\theta_{1}, \ldots, \theta_{p}\right)^{t}$ and $\boldsymbol{\phi}=\left(\phi_{1}, \ldots, \phi_{n}\right)^{t}$. For the moment we assume that the two models are nested, that is the statistics $s_{1}(\boldsymbol{y}), \ldots, s_{p}(\boldsymbol{y})$ are the same in models $m_{1}$ and $m_{2}$. In the next subsection we present a more general approach for non-nested models. Following Bayes theorem the so-called evidence for each model can be calculated using

$$
p\left(\boldsymbol{y} \mid m_{1}\right)=\frac{f\left(\boldsymbol{y} \mid \boldsymbol{\theta}^{\prime}\right) p\left(\boldsymbol{\theta}^{\prime}\right)}{p\left(\boldsymbol{\theta}^{\prime} \mid \boldsymbol{y}\right)}, \quad \forall \boldsymbol{\theta}^{\prime},
$$

for model $m_{1}$, and

$$
\begin{aligned}
p\left(\boldsymbol{y} \mid m_{2}\right) & =\frac{f\left(\boldsymbol{y} \mid \boldsymbol{\theta}, \boldsymbol{\phi}, \mu_{\phi}, \sigma_{\phi}^{2}\right) p(\boldsymbol{\theta}) p\left(\phi \mid \mu_{\phi}, \sigma_{\phi}^{2}\right) p\left(\mu_{\phi}\right) p\left(\sigma_{\phi}^{2}\right)}{p\left(\boldsymbol{\theta}, \boldsymbol{\phi}, \mu_{\phi}, \sigma_{\phi}^{2} \mid \boldsymbol{y}\right)}, \quad \forall \boldsymbol{\theta}, \boldsymbol{\phi}, \mu_{\phi}, \sigma_{\phi}^{2}, \\
& =\frac{f\left(\boldsymbol{y} \mid \boldsymbol{\theta}, \mu_{\phi}, \sigma_{\phi}^{2}\right) p(\boldsymbol{\theta}) p\left(\mu_{\phi}\right) p\left(\sigma_{\phi}^{2}\right)}{p\left(\boldsymbol{\theta}, \mu_{\phi}, \sigma_{\phi}^{2} \mid \boldsymbol{y}\right)}, \quad \forall \boldsymbol{\theta}, \mu_{\phi}, \sigma_{\phi}^{2}
\end{aligned}
$$

for model $m_{2}$.

The term $f\left(\boldsymbol{y} \mid \boldsymbol{\theta}, \mu_{\phi}, \sigma_{\phi}^{2}\right)$ denotes the marginal likelihood from model $m_{2}$, where the 
random effects $\phi$ have been marginalized, i.e.

$$
\begin{aligned}
f\left(\boldsymbol{y} \mid \boldsymbol{\theta}, \mu_{\phi}, \sigma_{\phi}^{2}\right) & =\int \frac{\exp \left\{\boldsymbol{\theta}^{t} s(\boldsymbol{y})+\boldsymbol{\phi}^{t} t(\boldsymbol{y})\right\}}{\kappa(\boldsymbol{\theta}, \boldsymbol{\phi})} \cdot p\left(\phi \mid \mu_{\phi}, \sigma_{\phi}^{2}\right) \mathrm{d} \boldsymbol{\phi} \\
& \approx \frac{\exp \left\{\boldsymbol{\theta}^{t} s(\boldsymbol{y})\right\}}{\kappa(\boldsymbol{\theta}, \widehat{\boldsymbol{\phi}})} \widehat{f}_{\text {Laplace }}\left(\boldsymbol{y} \mid \widehat{\boldsymbol{\phi}}, \mu_{\phi}, \sigma_{\phi}^{2}\right) .
\end{aligned}
$$

The approximation in equation (12) is achieved using a Laplace approximation around the point $\widehat{\phi}$. Details of this approximation are given in Section A of the appendix.

The Bayes factor of model $m_{2}$ against model $m_{1}$ is then defined as the ratio of (11) and (10), i.e.

$$
\mathrm{BF}_{21}=\frac{p\left(\boldsymbol{y} \mid m_{2}\right)}{p\left(\boldsymbol{y} \mid m_{1}\right)}=\frac{f\left(\boldsymbol{y} \mid \boldsymbol{\theta}, \mu_{\phi}, \sigma_{\phi}^{2}\right)}{f\left(\boldsymbol{y} \mid \boldsymbol{\theta}^{\prime}\right)} \cdot \frac{p(\boldsymbol{\theta}) p\left(\mu_{\phi}\right) p\left(\sigma_{\phi}^{2}\right)}{p\left(\boldsymbol{\theta}^{\prime}\right)} \cdot \frac{p\left(\boldsymbol{\theta}^{\prime} \mid \boldsymbol{y}\right)}{p\left(\boldsymbol{\theta}, \mu_{\phi}, \sigma_{\phi}^{2} \mid \boldsymbol{y}\right)} .
$$

Applying the approximation from equation (12) to (13), and plugging in estimates for the posterior densities

$$
p\left(\boldsymbol{\theta}^{\prime} \mid \boldsymbol{y}\right) \approx \hat{p}\left(\boldsymbol{\theta}^{\prime} \mid \boldsymbol{y}\right) \quad \text { and } \quad p\left(\boldsymbol{\theta}, \mu_{\phi}, \sigma_{\phi}^{2} \mid \boldsymbol{y}\right) \approx \widehat{p}\left(\boldsymbol{\theta}, \mu_{\phi}, \sigma_{\phi}^{2} \mid \boldsymbol{y}\right)
$$

leads to

$$
\mathrm{BF}_{21} \approx \frac{\exp \left\{\boldsymbol{\theta}^{t} s(\boldsymbol{y})\right\} \widehat{f}_{\text {Laplace }}\left(\boldsymbol{y} \mid \widehat{\boldsymbol{\phi}}, \mu_{\phi}, \sigma_{\phi}^{2}\right)}{\exp \left\{\boldsymbol{\theta}^{\prime t} s^{\prime}(\boldsymbol{y})\right\}} \cdot \frac{\kappa\left(\boldsymbol{\theta}^{\prime}\right)}{\kappa(\boldsymbol{\theta}, \widehat{\boldsymbol{\phi}})} \cdot \frac{p(\boldsymbol{\theta}) p\left(\mu_{\phi}\right) p\left(\sigma_{\phi}^{2}\right)}{p\left(\boldsymbol{\theta}^{\prime}\right)} \cdot \frac{\widehat{p}\left(\boldsymbol{\theta}^{\prime} \mid \boldsymbol{y}\right)}{\widehat{p}\left(\boldsymbol{\theta}, \mu_{\phi}, \sigma_{\phi}^{2} \mid \boldsymbol{y}\right)} .
$$

The ratio of the two normalizing constants $\kappa\left(\boldsymbol{\theta}^{\prime}\right) / \kappa(\boldsymbol{\theta}, \widehat{\boldsymbol{\phi}})$ in (15) is estimated using a path sampling approach (Gelman and Meng, 1998), which is similarly used by Caimo and Friel (2013). Consider

$$
\kappa(\boldsymbol{\theta}(g), \boldsymbol{\phi}(g))
$$

where

$$
\begin{aligned}
& \boldsymbol{\theta}(g)=(1-g) \cdot \boldsymbol{\theta}^{\prime}+g \cdot\left[\begin{array}{l}
0 \\
\boldsymbol{\theta}
\end{array}\right] \text { and } \\
& \boldsymbol{\phi}(g)=g \cdot \boldsymbol{\phi}
\end{aligned}
$$

for $g \in[0,1]$. So by construction

$$
(\boldsymbol{\theta}(0), \boldsymbol{\phi}(0))=\left(\boldsymbol{\theta}^{\prime}, \mathbf{0}\right) \text { and } \quad(\boldsymbol{\theta}(1), \boldsymbol{\phi}(1))=\left(\left[\begin{array}{l}
0 \\
\boldsymbol{\theta}
\end{array}\right], \boldsymbol{\phi}\right) \cdot 1
$$

Then thermodynamic integration (or so-called path sampling) can be used to estimate

$$
\log \left\{\frac{\kappa\left(\boldsymbol{\theta}^{\prime}\right)}{\kappa(\boldsymbol{\theta}, \boldsymbol{\phi})}\right\}=\int_{0}^{1} \mathbb{E}_{\boldsymbol{Y} \mid \boldsymbol{\theta}(g), \boldsymbol{\phi}(g)}\left[\left(\boldsymbol{\theta}^{\prime}-\left[\begin{array}{l}
0 \\
\boldsymbol{\theta}
\end{array}\right]\right)^{t} s^{\prime}(\boldsymbol{Y})+(-\boldsymbol{\phi})^{t} t(\boldsymbol{Y})\right] \mathrm{d} g .
$$

\footnotetext{
${ }^{1}$ Note that the additional 0 entry is necessary because the mixed effects model contains no parameter for the edges statistic. The edge effect is captured in the mean value $\mu_{\phi}$ of the nodal random effects. The missing edges statistics is also the difference between $s^{\prime}(\boldsymbol{y})$ and $s(\boldsymbol{y})$.
} 
Consider discretising $g \in[0,1]$ as $\left(g_{0}=0, \ldots, g_{i}=\frac{i}{I}, \ldots, g_{I}=1\right)$. Then we approximate

$$
\begin{aligned}
E_{i} & :=\mathbb{E}_{\boldsymbol{Y} \mid \boldsymbol{\theta}\left(g_{i}\right), \boldsymbol{\phi}\left(g_{i}\right)}\left[\left(\boldsymbol{\theta}^{\prime}-\left[\begin{array}{c}
0 \\
\boldsymbol{\theta}
\end{array}\right]\right)^{t} s^{\prime}(\boldsymbol{Y})+(-\boldsymbol{\phi})^{t} t(\boldsymbol{Y})\right] \\
& \approx \frac{1}{N} \sum_{j=1}^{N}\left[\left(\boldsymbol{\theta}^{\prime}-\left[\begin{array}{l}
0 \\
\boldsymbol{\theta}
\end{array}\right]\right)^{t} s^{\prime}\left(\boldsymbol{y}^{(j)}\right)+(-\boldsymbol{\phi})^{t} t\left(\boldsymbol{y}^{(j)}\right)\right],
\end{aligned}
$$

where the networks $\boldsymbol{y}^{(j)}$ are drawn from $f\left(\boldsymbol{y} \mid \boldsymbol{\theta}\left(g_{i}\right), \boldsymbol{\phi}\left(g_{i}\right)\right)$, for $j=1, \ldots, N$. Then we use a trapezoidal rule to numerically integrate

$$
\log \left\{\frac{\kappa\left(\boldsymbol{\theta}^{\prime}\right)}{\kappa(\boldsymbol{\theta}, \boldsymbol{\phi})}\right\}=\sum_{i=1}^{I-1}\left(g_{i+1}-g_{i}\right) \cdot\left(\frac{E_{i+1}+E_{i}}{2}\right) .
$$

The path sampling routine can easily be parallelised because the evaluations at the individual grid points of $g$ do not depend on each other.

The Bayes factor in equation (15) is evaluated using the posterior mean values for the parameters $\boldsymbol{\theta}, \boldsymbol{\theta}^{\prime}, \mu_{\phi}$ and also for $\widehat{\boldsymbol{\phi}}$. For $\sigma_{\phi}^{2}$ we plug in the mean of the logarithmized values and transform it back onto the scale of $\sigma_{\phi}^{2}$, because the posterior density of $\sigma_{\phi}^{2}$ is not symmetric.

For reasons of simplicity the posterior density estimates (14) are estimated assuming asymptotic normality, again using $\log \left(\sigma_{\phi}^{2}\right)$. For the data examples in the next section this assumption seems to be reasonable when looking at the plotted posterior density estimates. Furthermore, the individual contributions of the different components of the Bayes factor calculation suggest that at least in these cases the posterior density estimates play a minor part compared to the other components. If this assumption is violated this step in the algorithm can be changed.

\subsection{Bayes Factor for Non-Nested Models}

The extension of the Bayes factor to allow for a comparison of non-nested models is relatively straight-forward. Let $m_{a}$ and $m_{b}$ be two not necessarily nested Bayesian Exponential Random Graph Models, with or without random effects. We denote the corresponding parameter vectors for each model with $\gamma_{a}$ and $\gamma_{b}$, respectively. The Bayes factor is defined through

$$
\mathrm{BF}_{b a}^{\text {non-nested }}=\frac{p\left(\boldsymbol{y} \mid m_{b}\right)}{p\left(\boldsymbol{y} \mid m_{a}\right)}=\frac{f\left(\boldsymbol{y} \mid \boldsymbol{\gamma}_{b}\right)}{f\left(\boldsymbol{y} \mid \boldsymbol{\gamma}_{a}\right)} \cdot \frac{p\left(\boldsymbol{\gamma}_{b}\right)}{p\left(\boldsymbol{\gamma}_{a}\right)} \cdot \frac{p\left(\boldsymbol{\gamma}_{a} \mid \boldsymbol{y}\right)}{p\left(\boldsymbol{\gamma}_{b} \mid \boldsymbol{y}\right)} .
$$

We distinguish three model setups. First (case 1), the model has fixed effects and nodal random effects, i.e. $\boldsymbol{\gamma}=\left(\boldsymbol{\theta}^{t}, \boldsymbol{\phi}^{t}\right)^{t}$. Second (case 2), the model has random nodal effects only, i.e. a classical $p_{2}$ model (see van Duijn et al., 2004) with parameters $\gamma=\phi$. Third (case 3), the model is a regular Exponential Random Graph Model without nodal random 
effects, i.e. the parameters result to $\boldsymbol{\gamma}=\boldsymbol{\theta}$. If the model contains random effects we use a Laplace approximation for the corresponding likelihood component $f(\boldsymbol{y} \mid \boldsymbol{\gamma})$ as in equation (12). The components of the approximated Bayes factor in equation (15) concerning prior and estimated posterior densities remain the same as in equation (15), again depending on whether each model contains nodal random effects or not. The evidence for each model in (16) can be approximated by

$$
p(\boldsymbol{y} \mid m) \approx \begin{cases}\frac{\exp \left\{\boldsymbol{\theta}^{t} s(\boldsymbol{y})\right\} \widehat{f}_{\text {Laplace }}\left(\boldsymbol{y} \mid \widehat{\boldsymbol{\phi}}, \mu_{\phi}, \sigma_{\phi}^{2}\right)}{\kappa(\boldsymbol{\theta}, \widehat{\boldsymbol{\phi}})} \cdot \frac{p(\boldsymbol{\theta}) p\left(\mu_{\phi}\right) p\left(\sigma_{\phi}^{2}\right)}{\widehat{p}\left(\boldsymbol{\theta}, \mu_{\phi}, \sigma_{\phi}^{2} \mid \boldsymbol{y}\right)}, & \text { (case 1), } \\ \frac{\widehat{f}_{\text {Laplace }}\left(\boldsymbol{y} \mid \widehat{\boldsymbol{\phi}}, \mu_{\phi}, \sigma_{\phi}^{2}\right)}{\kappa(\widehat{\boldsymbol{\phi}})} \cdot \frac{p\left(\mu_{\phi}\right) p\left(\sigma_{\phi}^{2}\right)}{\widehat{p}\left(\mu_{\phi}, \sigma_{\phi}^{2} \mid \boldsymbol{y}\right)}, & \text { (case 2), } \\ \frac{\exp \left\{\boldsymbol{\theta}^{t} s(\boldsymbol{y})\right\}}{\kappa(\boldsymbol{\theta})} \cdot \frac{p(\boldsymbol{\theta})}{\widehat{p}(\boldsymbol{\theta} \mid \boldsymbol{y})}, & \text { (case 3). }\end{cases}
$$

The major change concerns the estimation of the ratio of the two normalizing constants in (15) via path sampling. Instead of estimating the ratio directly we estimate two ratios,

$$
\kappa(\mathbf{0}) / \kappa\left(\boldsymbol{\gamma}_{a}\right) \text { and } \kappa(\mathbf{0}) / \kappa\left(\boldsymbol{\gamma}_{b}\right),
$$

via path sampling, where $\kappa(\mathbf{0})$ is the normalizing constant of a null model where all parameters are set to 0 . The path sampling works in the same way as described before, but we substitute one of the models by the null model. By dividing the two estimated ratios, the term $\kappa(\mathbf{0})$ cancels out and we obtain an estimate of $\kappa\left(\gamma_{a}\right) / \kappa\left(\gamma_{b}\right)$. It should be clear this approach takes almost double the amount of time (or cores) to estimate the ratio of normalizing constants as in the nested case where we need only one instance of path sampling. This suggests to use the Bayes factor (15) if the models are nested.

\section{Examples}

\subsection{Data Examples}

\subsubsection{Zachary's Karate Club Network}

As a first data example we employ Zachary's karate club network (Zachary, 1977) which is a very well known data set often used in network analysis. The undirected 34 node network represents the friendships among members of a university karate club. Figure 2 shows a plot of this network graph. It is evident that there are only some nodes with a very high degree (no. 1,33, and 34) while the majority of the remaining vertices has only two to four links. If there are no additional nodal attributes available, that might explain some differences between the actors, like for example status in the club (trainer, student, etc.), the assumption of vertex homogeneity in a standard ERGM appears to be at least questionable. 

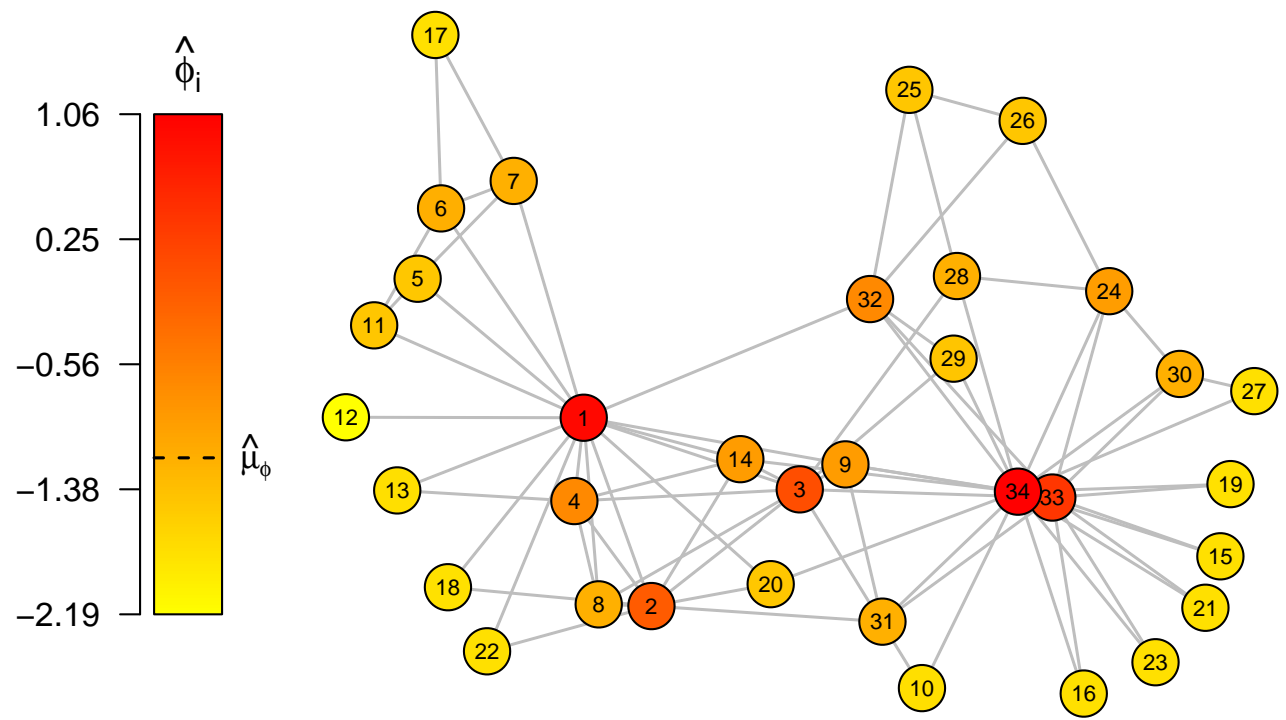

Figure 2 Zachary's Karate Club Graph. Vertices are coloured by their estimated nodal effect $\widehat{\phi}_{i}$ (posterior mean), $i=1, \ldots, n$. Vertices with a high nodal effect are darker in orange/red.

As a first step, we fitted two different models to the data: a standard ERGM with edges and triangles as sufficient statistics, and a model with nodal random effects and the triangle statistic. These two models are nested.

For the model fitting tasks we used the Bergm package (Caimo and Friel, 2014) and our extension of the Bergm routines, respectively. With 1,000 burn-in iterations, 30,000 main iterations, and 3,000 auxiliary iterations for the network simulation in each MCMC step, the computation of the fixed model took about two minutes on a $2.1 \mathrm{Ghz}$ processor, the mixed model needed about one hour and forty minutes. Using 3,000 auxiliary iterations should be large enough because we have 561 possible ties in the network. Again, we refer to the results of Everitt (2012).

Figure 3 shows the results for the fixed model with edges and triangular effect only.

Figure 4 shows the results for the mixed model with nodal random and triangular effects for the karate club data.

Table 1 shows the resulting posterior estimates for both models.

The vertices of the karate network in Figure 2 are coloured according to their estimated nodal effect $\widehat{\phi}_{i}, i=1, \ldots, n$. As an estimate we use the corresponding posterior mean of each parameter $\phi_{i}$. Darker coloured vertices (orange/red) correspond to those with a high nodal value. By using such a colouring scheme we are able to visualise the variation in the nodal effects. In addition, we can 

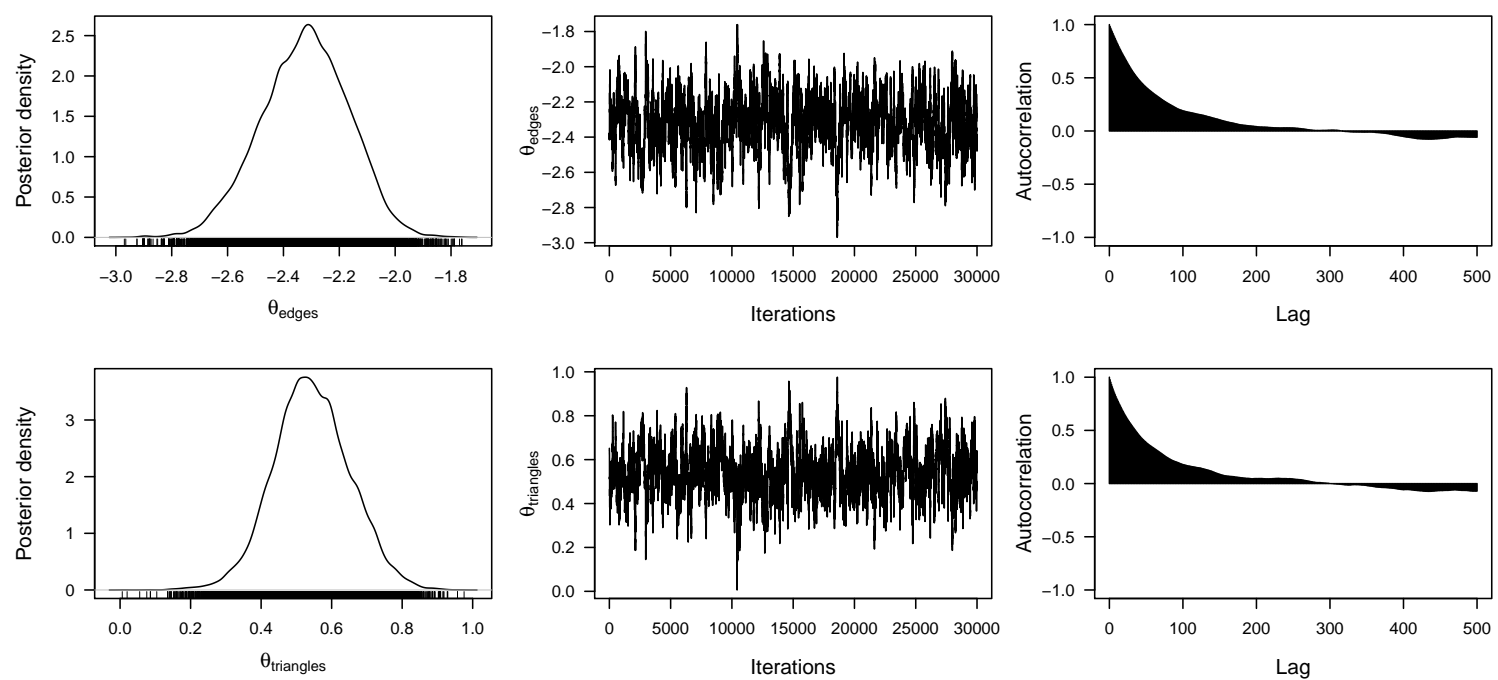

Figure 3 Posterior densities, trace plots, and autocorrelation for the fixed model with edges and triangular effect for the karate club data.
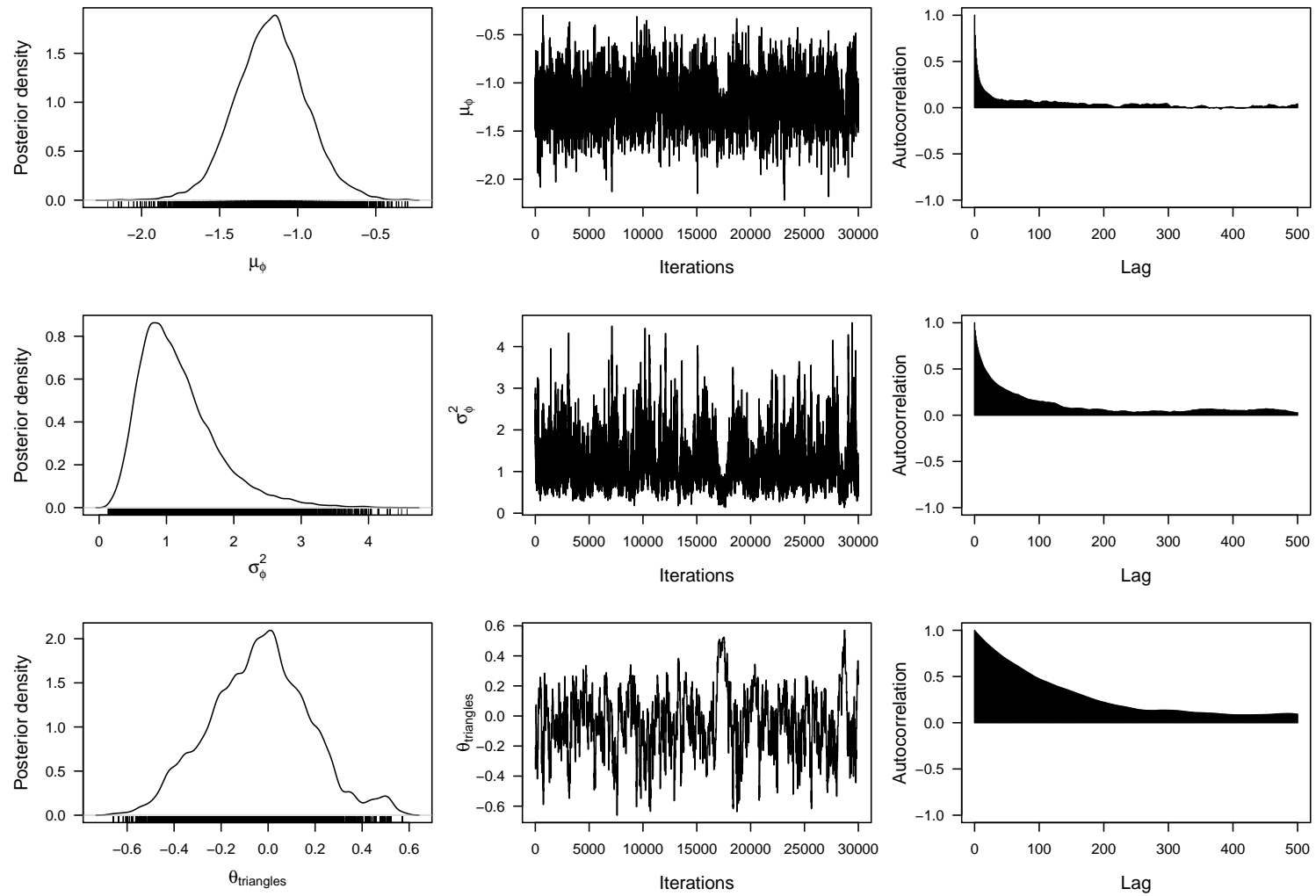

Figure 4 Posterior densities, trace plots, and autocorrelation for the mixed model with nodal random and triangular effects for the karate club data.

identify important nodes in the network based on the estimated nodal effects.

Figure 5 shows estimates for the posterior densities for both models simultaneously to allow for a visual comparison. 
Table 1 Model fitting results for the karate club data. The fixed model contains edges and triangles, and the mixed model triangles and nodal random effects.

Model type Parameter Post. mean Post. Sd. Acceptance rate Note

\begin{tabular}{llllll}
\hline \hline \multirow{3}{*}{ fixed } & $\theta_{\text {edges }}$ & -2.32 & 0.16 & & \\
& $\theta_{\text {triangles }}$ & 0.54 & 0.11 & 0.43 & \\
\hline \multirow{3}{*}{ mixed } & $\mu_{\phi}$ & -1.17 & 0.22 & 0.26 & $*$ \\
& $\sigma_{\phi}^{2}$ & 1.05 & 0.58 & 0.54 & $* 09$ \\
& $\theta_{\text {triangles }}$ & -0.04 & 0.21 & 0.09 & \\
\hline
\end{tabular}

* For $\sigma_{\phi}^{2}$ the posterior mean is calculated based on the logarithmized values and then transformed back to the scale of $\sigma_{\phi}^{2}$ (this leads to the geometric mean) due to the nonsymmetric posterior density in this case.
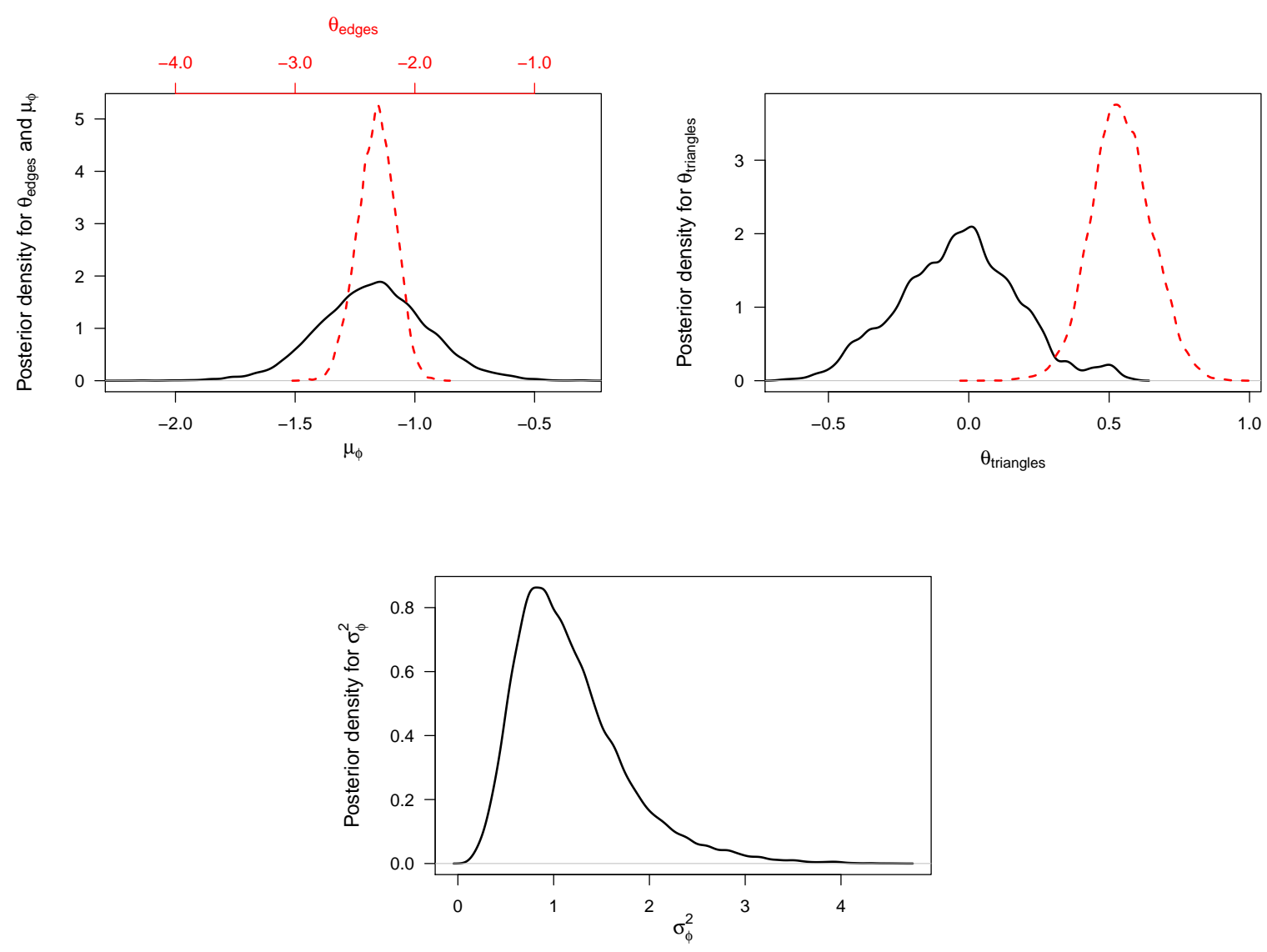

Figure 5 Posterior densities for the model with edges and triangular effect (red dashed lines) and nodal random and triangular effects (black solid lines) for the karate club data.

What is evident from the estimated posterior densities is the difference for the triangular effect in both models in the upper right plot of Figure 5. When not accounting for nodal heterogeneity this effect is clearly positive compared to the mixed model where the 
posterior support clearly comprises zero. For the parameter $\theta_{\text {edges }}$ associated with the edges statistic and $\mu_{\phi}$ in the mixed model there is no big difference between both models concerning the location of the posterior (when comparing $\theta_{\text {edges }}$ to $2 \cdot \mu_{\phi}$; note the different axis annotations).
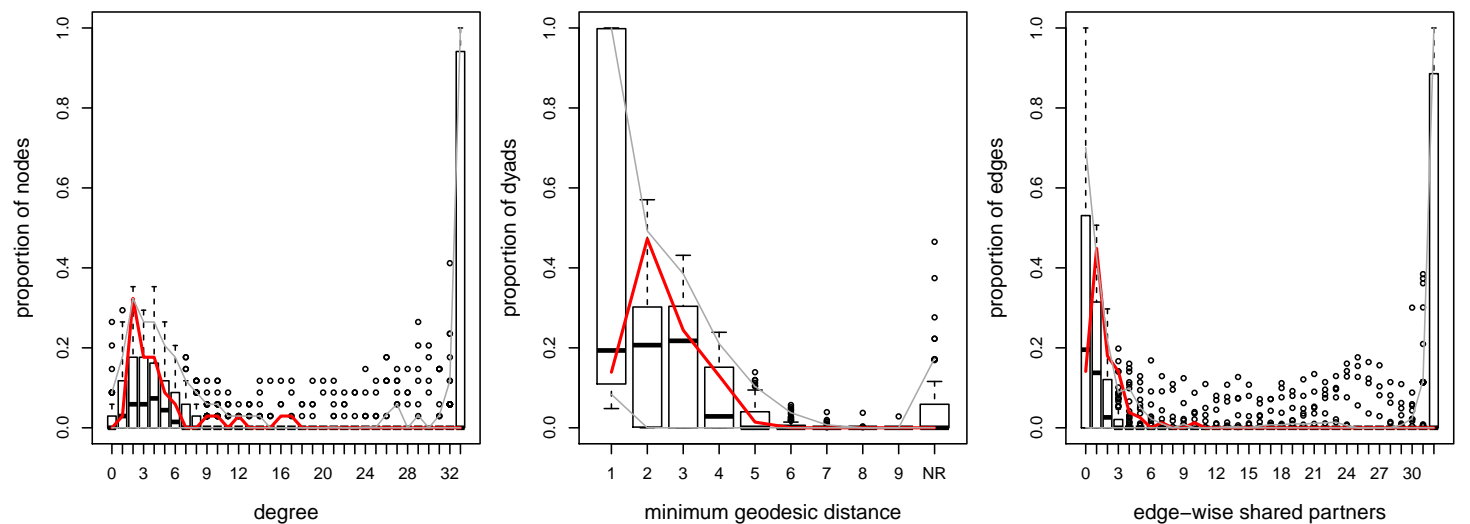

Figure 6 Bayesian goodness-of-fit diagnostics for the fixed model with edges and triangle effect for the karate club data. Bold red line corresponds to original dataset.
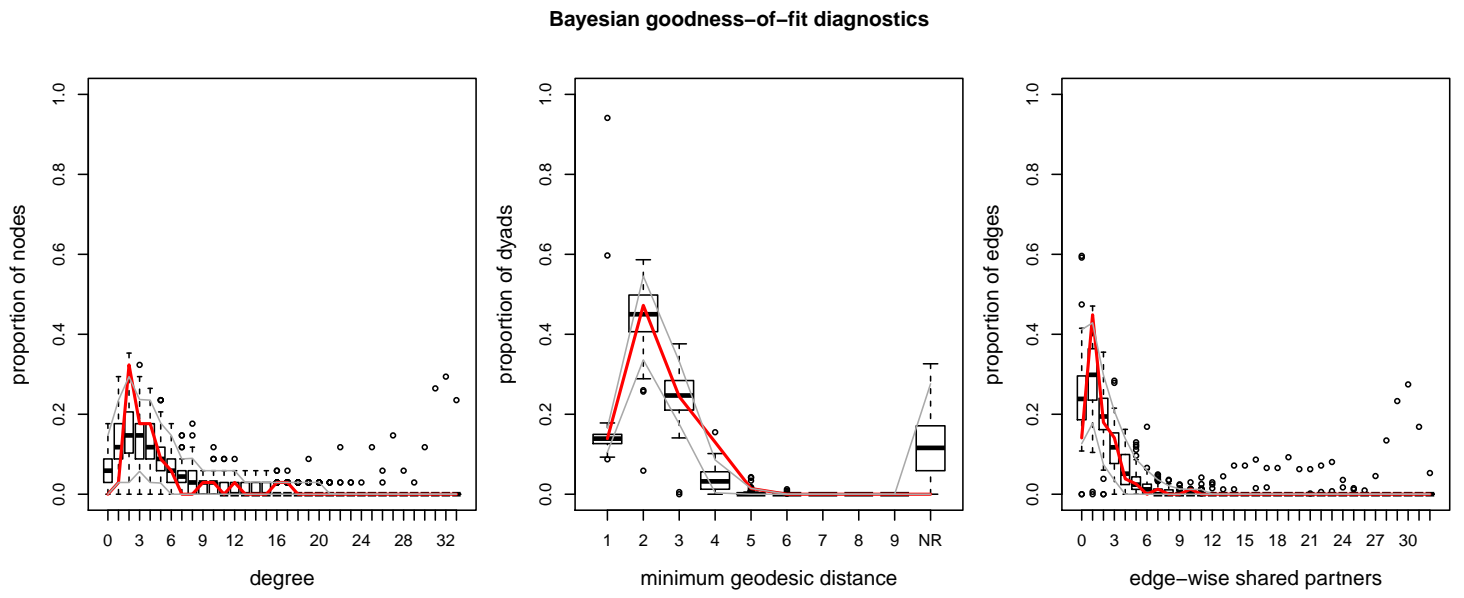

Figure 7 Bayesian goodness-of-fit diagnostics for the mixed model with triangle and nodal random effects for the karate club data. Bold red line corresponds to original dataset.

Figure 6 and Figure 7 show Bayesian goodness-of-fit plots for both models. For each model we used 100 draws from the corresponding posterior and simulated a network for each of the posterior parameter combinations. Boxplots of the distributions of degree, minimum geodesic distance, and edge-wise shared partners for the resulting simulated networks are shown in the plots where the bold red line indicates the values of the original karate club network. If other aspects of the data are of interest in order to assess goodness-of-fit of a model, e.g., triad census (see, e.g., Caimo and Friel, 2011, Figure 15) the 
goodness-of-fit plots can be customized accordingly. For the fixed model with edges and triangle effect we see some problems in Figure 6 concerning especially the degree distribution and the edgewise-shared partner distribution. For some (but not all) of the resulting simulated networks we have a high proportion of nodes with degree 33, which corresponds to a full or an almost full network. The same applies to very high proportions of edges with 32 edge-wise shared partners. It is well known from the literature, see, for example, Lusher et al. (2013), Chatterjee and Diaconis (2013), and Schweinberger (2011), that an Exponential Random Graph Model containing only edges and triangles as statistics is problematic, i.e. we have degeneracy issues. In the Bayesian setting here the model with edges and triangles does not result in complete degeneracy as only some but not all of the resulting simulated networks are complete graphs, which coincides with the findings of (Caimo and Friel, 2011). For the simulation of each network we use 10000 iterations which is sufficiently large for a network with 34 nodes to assume convergence of the underlying chain.

For the mixed model with triangles and nodal random effects the resulting plots in Figure 7 look reasonable.

Especially the different effect of the triangular statistic in both models clearly illustrates the issue of model selection. After fitting the two competing models we computed a Bayes factor using the approach for nested models described in Section 3.2 to compare the model with nodal random effects to the one with structural effects only and tackle this issue.

The resulting estimated log Bayes factor is 453, which is huge. As explained in the previous section, there is some randomness involved in the procedure. Repeated calculation led to similarly huge values. This clearly indicates that the model with nodal random effects is preferable to the one without and is not surprising, because here a model with nodal heterogeneity appears much more realistic than one without.

Computing a single Bayes factor took about fourteen minutes using five 2.2 Ghz cores in parallel, with 10,000 iterations for the Laplace approximation, 1,000 grid points, 1,000 iterations at each point for the path sampling, and 3,000 iterations for each network simulation.

As second step of our analysis of the karate data we have used a model containing edges and the geometrically weighted edge-wise shared partner (GWESP) statistic

$$
e^{\theta_{\mathrm{dec}}} \sum_{i=1}^{n-2}\left\{1-\left(1-e^{-\theta_{\mathbf{d e c}}}\right)^{i}\right\} \mathbf{E P}_{i}(\boldsymbol{y})
$$

where $\operatorname{EP}_{i}(\boldsymbol{y})$ denotes the number of edges with $i$ shared partners, see Hunter (2007) for details. Adding this term to the model circumvents the degeneracy issue known from the edges and triangle model. Even though it can be shown that the term is equivalent to the alternating $k$-triangle statistic, see Hunter 
(2007), and Snijders et al. (2006), from a modelling point of view, both terms, GWESP and the alternating $k$-triangles are more complicated to interpret. By setting the decay parameter to a fixed value, in our case $\theta_{\text {dec }}=0.8$, the model is a regular, non-curved Exponential Random Graph Model (Hunter and Handcock, 2006).

In addition we have fitted a mixed model with nodal random effects and the GWESP statistic, again with a fixed decay parameter $\theta_{\mathrm{dec}}=0.8$, and a model containing only the nodal random effects, which is just the Bayesian version of a $p_{2}$ model (van Duijn et al., 2004). Table 2 shows the resulting posterior estimates for all three models.

Table 2 Model fitting results for the karate club data. The fixed model contains edges and geometrically weighted edge-wise shared partners (GWESP), and the mixed model GWESP and nodal random effects. For both models the decay parameter for GWESP is fixed at 0.8. The random model contains only the nodal random effects.

\begin{tabular}{llcccc}
\hline \multirow{2}{*}{ Model type } & Parameter & Post. mean & Post. Sd. & Acceptance rate & Note \\
\hline \hline \multirow{2}{*}{ fixed } & $\theta_{\text {edges }}$ & -2.99 & 0.24 & 0.37 & \\
& $\theta_{\text {gwesp }}$ & 0.63 & 0.11 & & \\
\hline \multirow{4}{*}{ mixed } & $\mu_{\phi}$ & -1.22 & 0.21 & 0.24 & $*$ \\
& $\sigma_{\phi}^{2}$ & 0.88 & 0.47 & 0.48 & $* .06$ \\
\hline \multirow{4}{*}{ random } & $\theta_{\text {gwesp }}^{2}$ & 0.08 & 0.13 & 0.06 & \\
& $\mu_{\phi}$ & -1.18 & 0.20 & 0.25 & $*$ \\
\hline
\end{tabular}

* For $\sigma_{\phi}^{2}$ the posterior mean is calculated based on the logarithmized values and then transformed back to the scale of $\sigma_{\phi}^{2}$ (this leads to the geometric mean) due to the nonsymmetric posterior density in this case.

Figure 8 shows the results for the fixed model with edges and geometrically weighted edge-wise shared partners effect. The resulting trace and autocorrelation plots for both parameters reveal that the Markov chain did not mix so well and this could have been alleviated by thinning the chain.

Figure 9 shows the results for the mixed model with geometrically weighted edge-wise shared partners (with fixed decay of 0.8) and nodal random effects for the karate club data. The trace plot and autocorrelation plot for $\theta_{\text {gwesp }}$ convey that the Markov chain did not mix as well for this parameter compared to the other two parameters.

Figure 10 shows the results for the model with random effects only for the karate club data. 

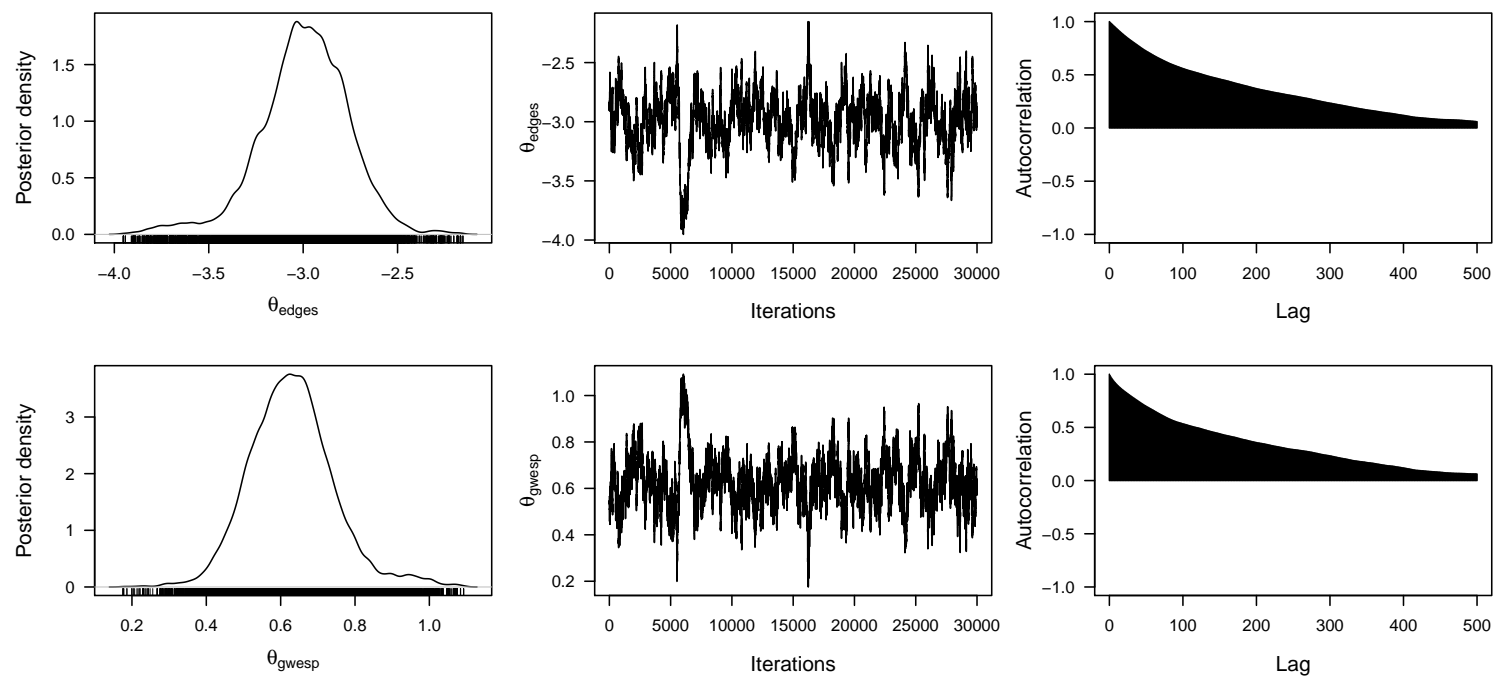

Figure 8 Posterior densities, trace plots, and autocorrelation for the fixed model with edges and geometrically weighted edge-wise shared partners (with fixed decay of 0.8 ) effect for the karate club data.
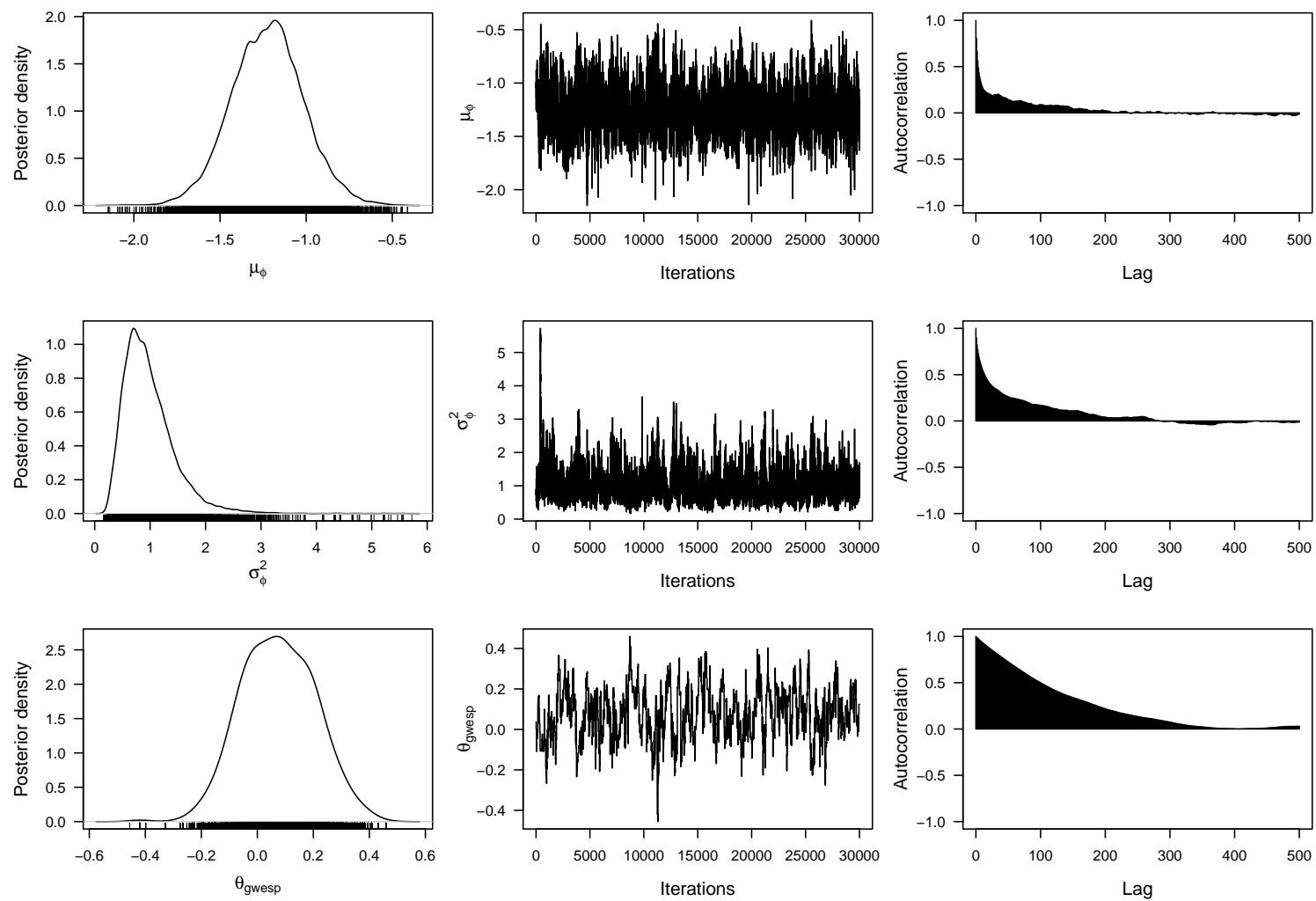

Figure 9 Posterior densities, trace plots, and autocorrelation for the mixed model with geometrically weighted edge-wise shared partners (with fixed decay of 0.8) and nodal random effects for the karate club data.

The posterior density for the parameter associated with the GWESP statistic is almost centred at zero in the mixed model, while in the fixed model the parameter is clearly positive. So we see a comparable behaviour as in the 

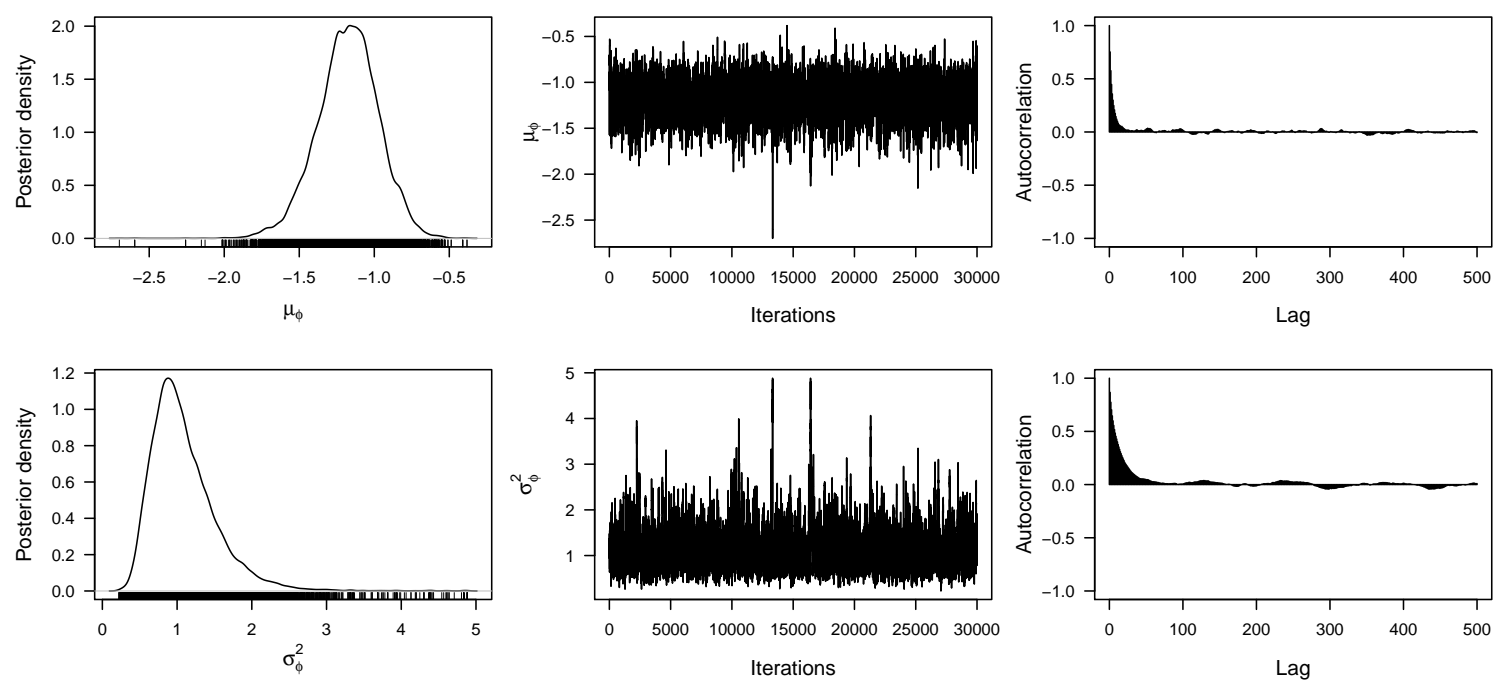

Figure 10 Posterior densities, trace plots, and autocorrelation for the model with nodal random effects for the karate club data.

models before for the triangle effect which is positive in the fixed model and becomes zero when nodal random effects are included in the model.

Figures 11 - 13 show the corresponding goodness-of-fit diagnostic plots for the three models. For the distribution of the minimum geodesic distance the model with edges and geometrically weighted edge-wise shared partner statistic seems to be better in capturing this property of the network than the two models with nodal random effects. In general, the resulting goodness-of-fit plots are comparable for all three models. Here we do not see any degenerate behaviour, i.e. there is no high proportion of nodes with degree 33 or a high proportion of edges with 32 shared partners which would be the case in a full graph.
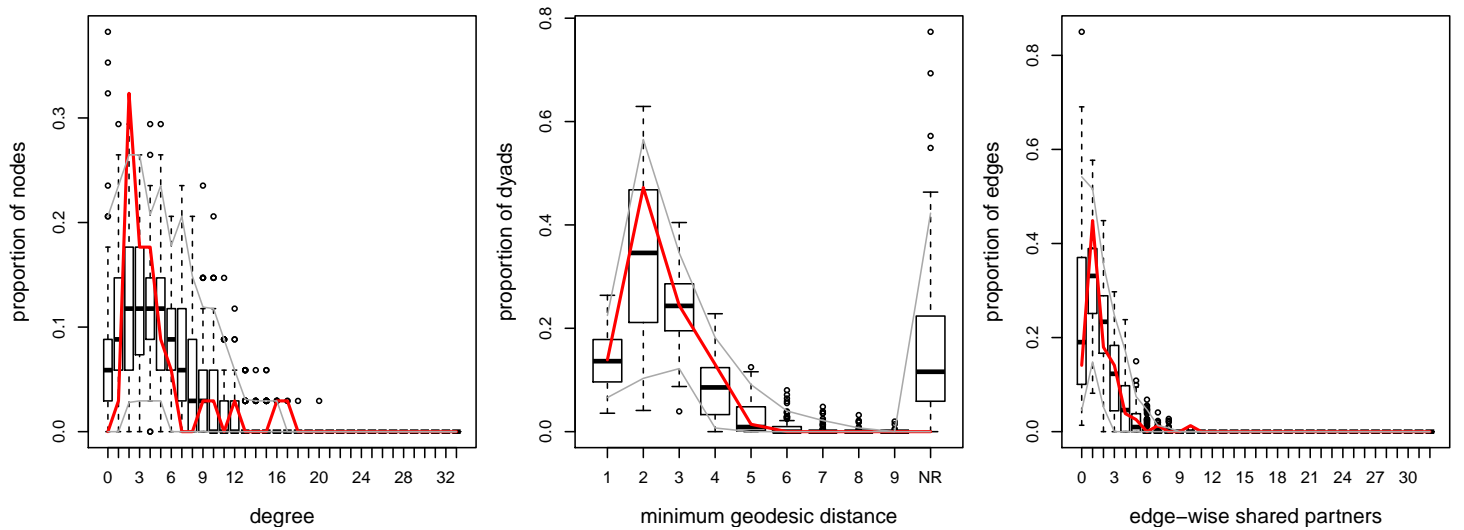

Figure 11 Bayesian goodness-of-fit diagnostics for the fixed model with edges and triangle effect for the karate club data. Bold red line corresponds to original dataset. 

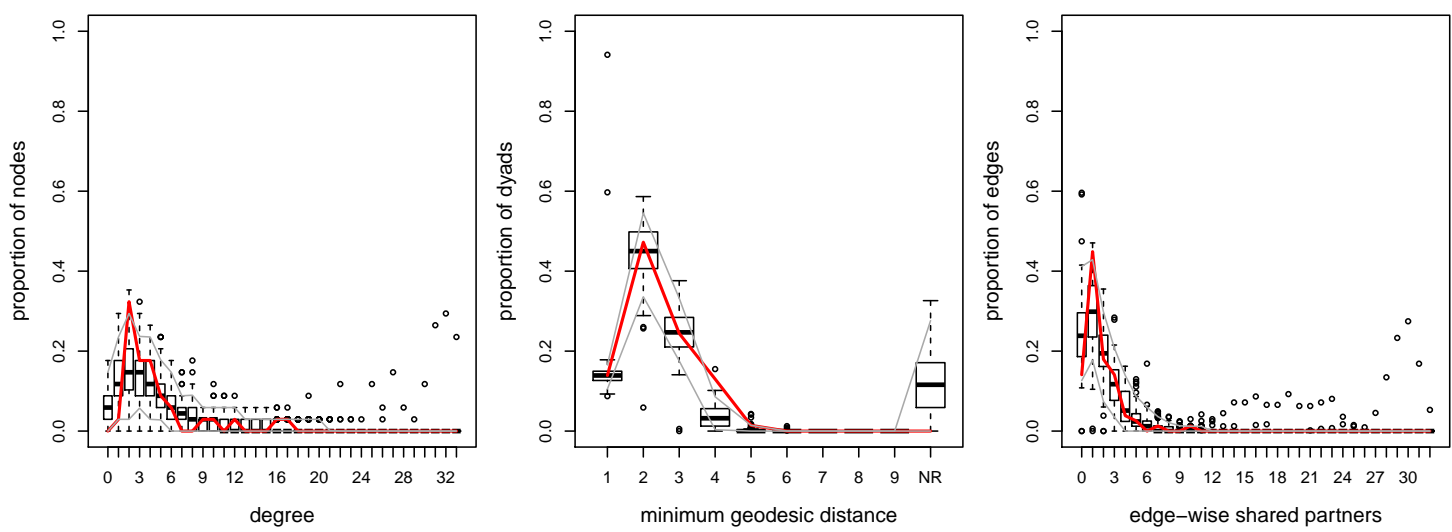

Figure 12 Bayesian goodness-of-fit diagnostics for the mixed model with triangle and nodal random effects for the karate club data. The bold red line corresponds to original dataset.
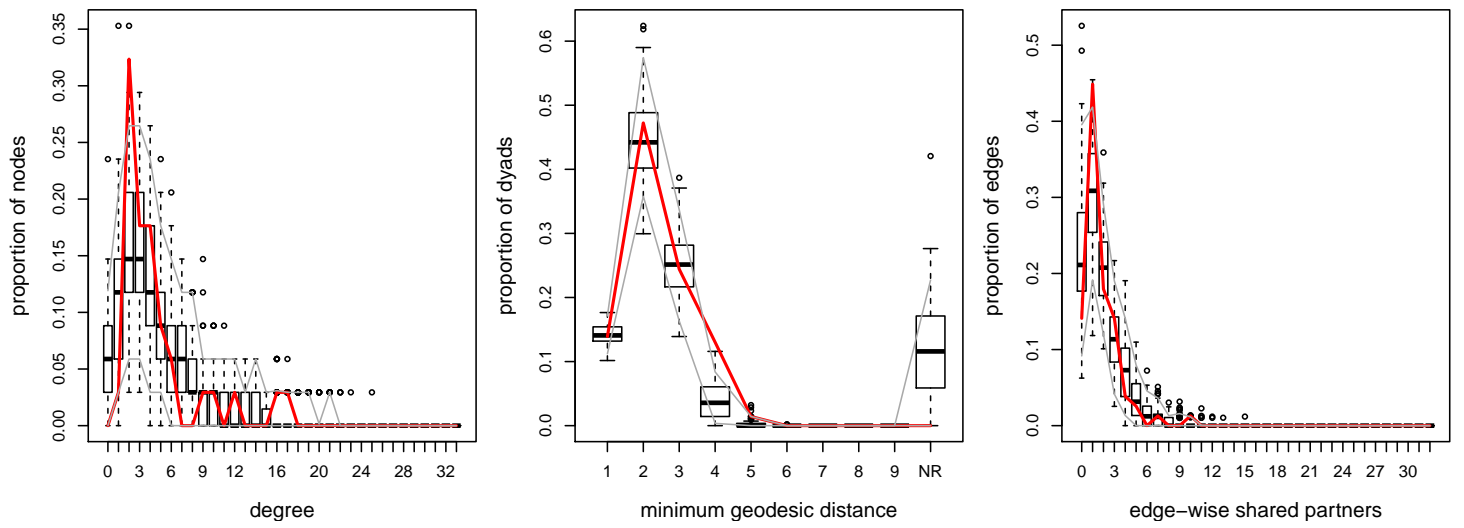

Figure 13 Bayesian goodness-of-fit diagnostics for model with random effects only for the karate club data. Bold red line corresponds to original dataset.

For model selection in the second part of the karate data analysis we have computed two Bayes factors. The resulting log Bayes factor for the nested comparison of the fixed model with edges and geometrically weighted edgewise shared partners against the mixed model with GWESP and nodal random effects is -0.41 . This value points into the direction of the fixed model, but is rather close to zero. So the conclusion here is that none of the two competing models is clearly better than the other. The second log Bayes factor for the non-nested comparison of the fixed model with edges and GWESP against the model with nodal random effects only is 3.83 , which is clearly in favour of the random effects model. So based on these values the model containing only nodal random effects would be preferred. 


\subsubsection{Kapferer Tailor Shop}

As a second data example we use the Kapferer network (Kapferer, 1972) which contains interactions among 39 workers in a tailor shop in Zambia. Figure 14 shows a plot of the network. The situation here is comparable to the karate data with only some high degree nodes.

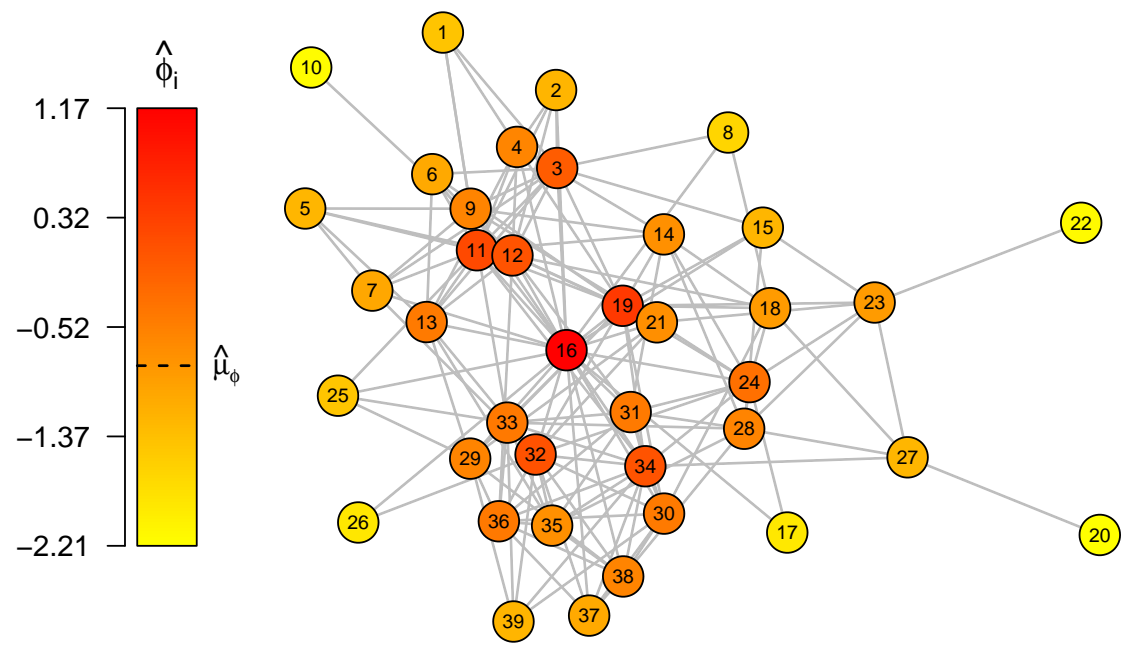

Figure 14 Kapferer Tailor Shop Network. Vertices are coloured by their estimated nodal effect $\widehat{\phi}_{i}$ (posterior mean), $i=1, \ldots, n$. Vertices with a high nodal effect are darker in orange/red.

Following Robins and Lusher (2013) we start with a fixed model containing edges, geometrically weighted degree with a fixed decay parameter of 0.7 and the 2-star statistic. The geometrically weighted degree statistic has the form

$$
e^{\theta_{\mathrm{dec}}} \sum_{i=1}^{n-1}\left\{1-\left(1-e^{-\theta_{\mathrm{dec}}}\right)^{i}\right\} \mathrm{D}_{i}(\boldsymbol{y}),
$$

where $\mathrm{D}_{i}(\boldsymbol{y})$ denotes the number of nodes with degree $i$, see Hunter (2007) for details. Again, we set the decay parameter to a fixed value $\theta_{\text {dec }}=0.7$ resulting in a regular, i.e. a non-curved ERGM (Hunter and Handcock, 2006). We fit a Bayesian Exponential Random Graph Model with 6 chains and adaptive direction sampling (Caimo and Friel, 2011) in order to improve mixing and obtain a more stable result than for a single chain model. The results for the overall chain are shown in Figure 15. Table 3 summarizes the results. The overall tendency of a small positive 2-star effect combined with a negative effect of the geometrically weighted degree corresponds to the suggestions of Robins and Lusher (2013) for modelling a heterogeneous degree distribution with some high degree nodes.

As competing model we fit a Bayesian ERGM with nodal random effects only, which is again basically the Bayesian analogue of a $p_{2}$ model. The results are shown in Figure 16 

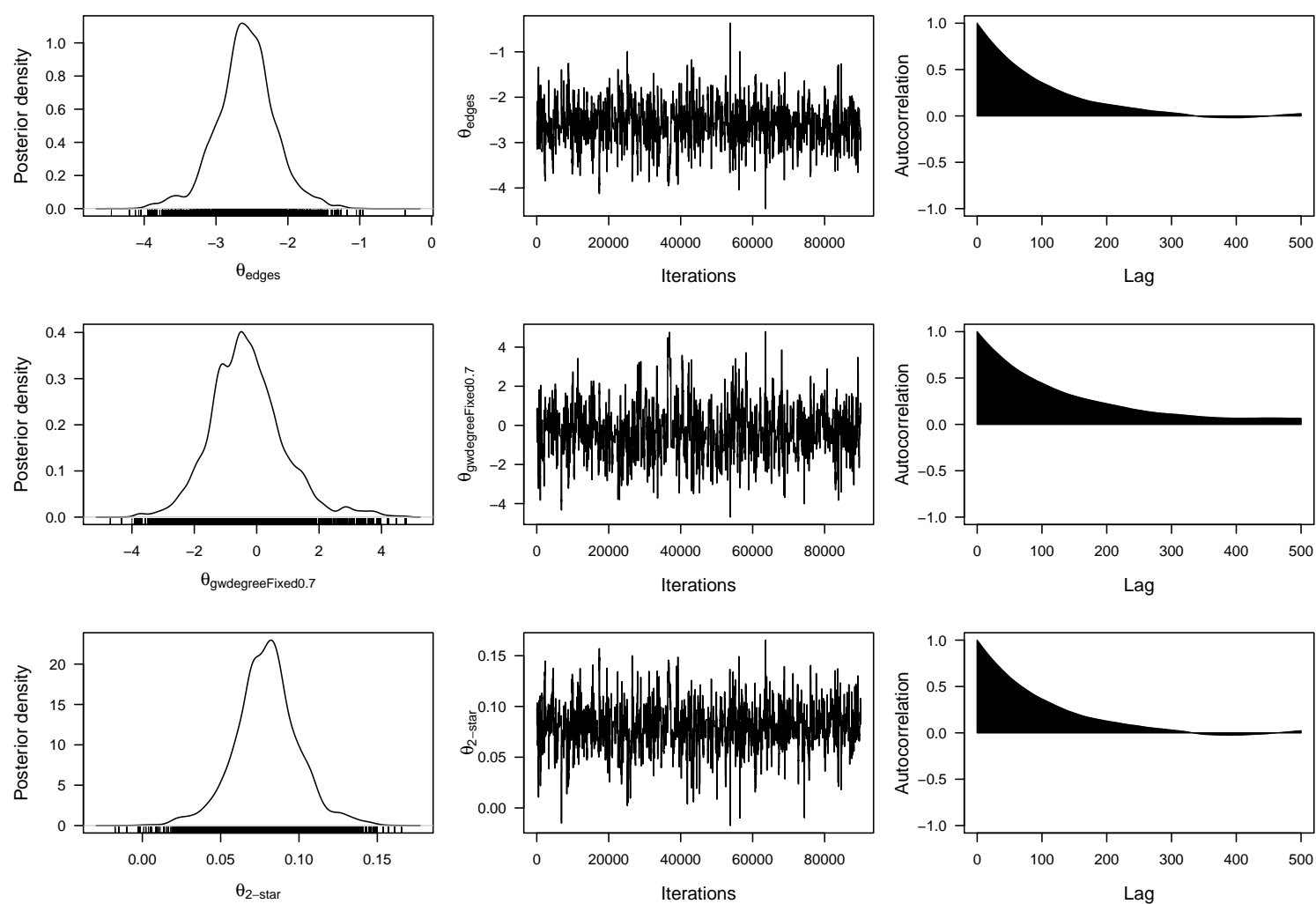

Figure 15 Posterior densities, trace plots, and autocorrelation for the fixed model with edges, geometrically weighted degree (with fixed decay of 0.7), and 2-star effect for the Kapferer data.

and Table 3.
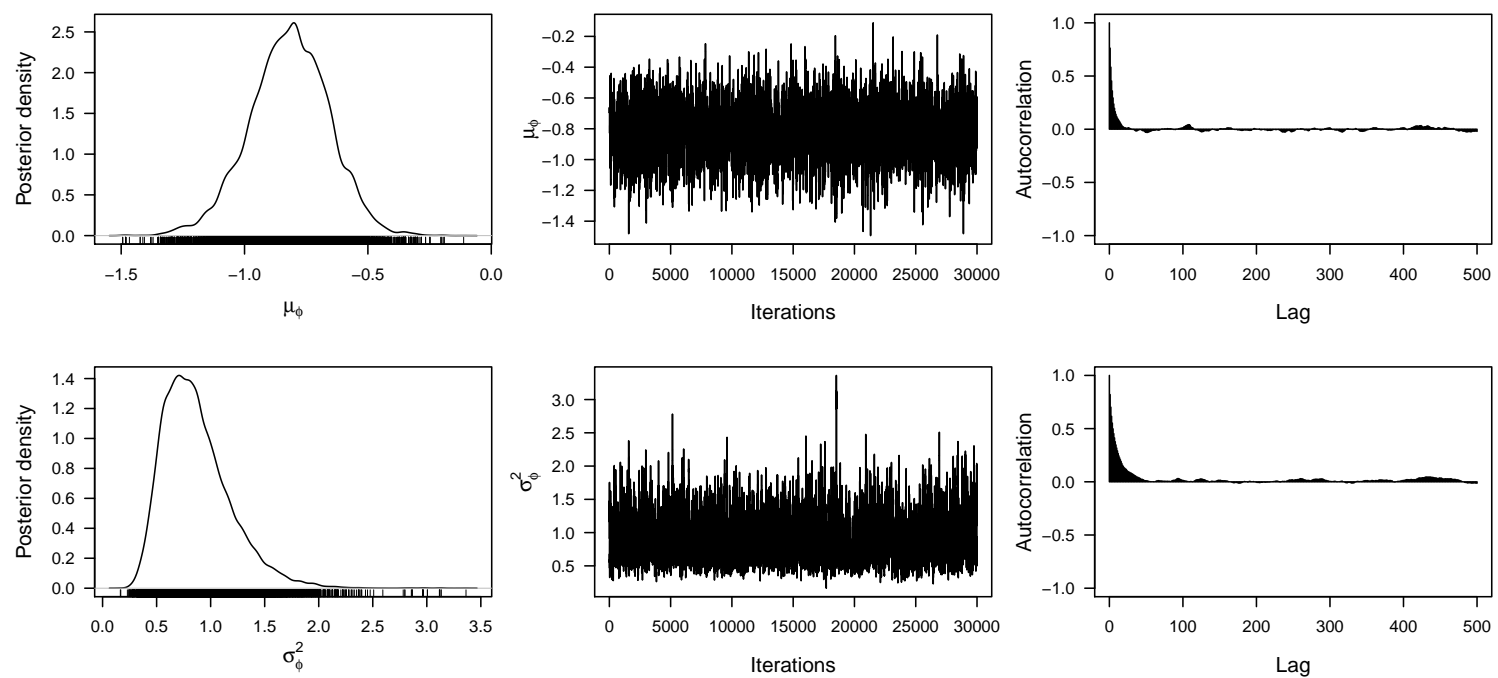

Figure 16 Posterior densities, trace plots, and autocorrelation for the model with nodal random effects for the Kapferer data.

Figure 17 and Figure 18 show Bayesian goodness-of-fit plots for both models. Similar to the goodness-of-fit plots in the previous data example, for each model we used 100 draws from the corresponding posterior and simulated a network for each of the posterior parameter combinations. Again, the plots show boxplots of the distributions 
Table 3 Model fitting results for the Kapferer data. The fixed model contains edges, geometrically weighted degree (with a fixed decay of 0.7), and the 2-star effect. The random model contains only the nodal random effects.

Model type Parameter Post. mean Post. Sd. Acceptance rate Note

\begin{tabular}{|c|c|c|c|c|c|}
\hline \multirow{3}{*}{ fixed } & $\theta_{\text {edges }}$ & -2.59 & 0.41 & \multirow{3}{*}{0.03} & \\
\hline & $\theta_{\text {gwdegreeFixed } 0.7}$ & -0.30 & 1.18 & & \\
\hline & $\theta_{2 \text {-star }}$ & 0.08 & 0.02 & & \\
\hline \multirow{2}{*}{ random } & $\mu_{\phi}$ & -0.82 & 0.16 & 0.22 & \\
\hline & $\sigma_{\phi}^{2}$ & 0.82 & 0.31 & 0.43 & $*$ \\
\hline
\end{tabular}

* For $\sigma_{\phi}^{2}$ the posterior mean is calculated based on the logarithmized values and then transformed back to the scale of $\sigma_{\phi}^{2}$ (this leads to the geometric mean) due to the nonsymmetric posterior density in this case.

of degree, minimum geodesic distance, and edge-wise shared partners for the resulting simulated networks. The bold red line indicates the values of the original Kapferer network. Degenerate behaviour did not occur here, i.e. there were no networks with high proportions of nodes with degree 38 or edges with 37 shared partners. Note that therefore the plots do not show the full distributions of the three measures to ease visual inspection. For the model with nodal random effects only the resulting simulated networks seem to be less extreme in the sense that the observed values for all three statistics are closer to the simulated ones and the boxplots are not as wide as for the fixed model.

The resulting log Bayes factor for the two non-nested models is 217, which is again quite big and supports the model with random effects only.
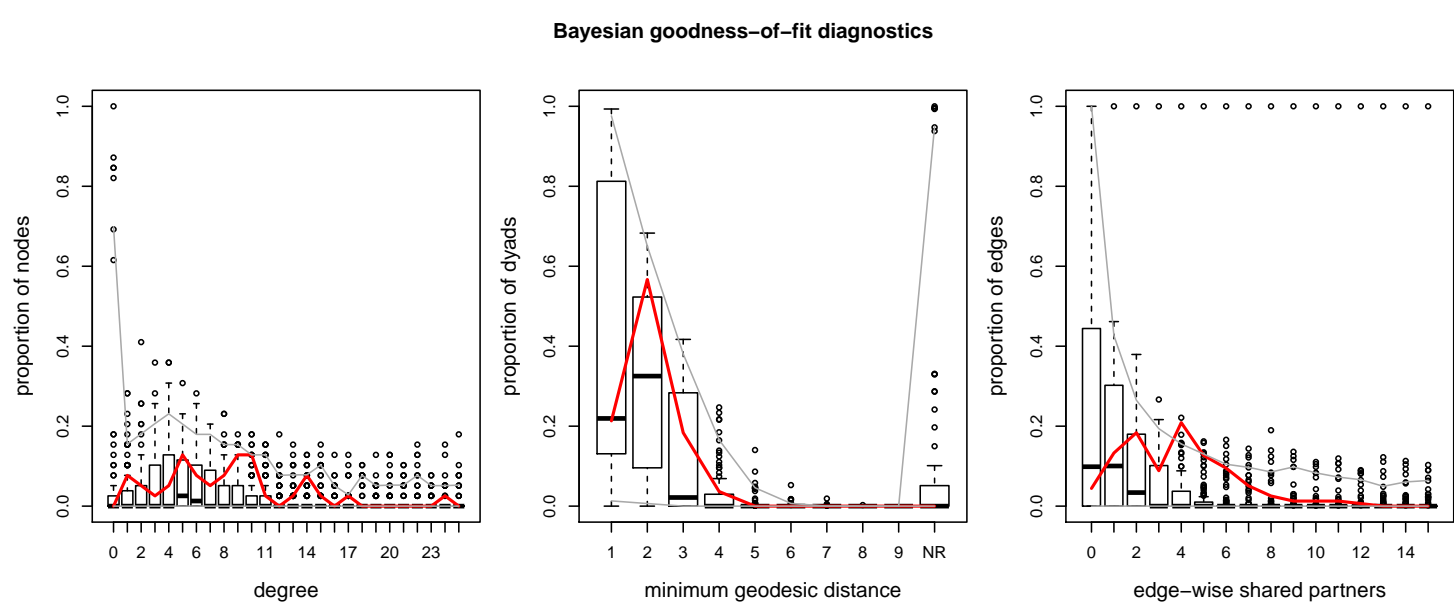

Figure 17 Bayesian goodness-of-fit diagnostics for the fixed model with edges, geometrically weighted degree (with fixed decay of 0.7), and 2-star effect for the Kapferer data. Bold red line corresponds to original dataset. 

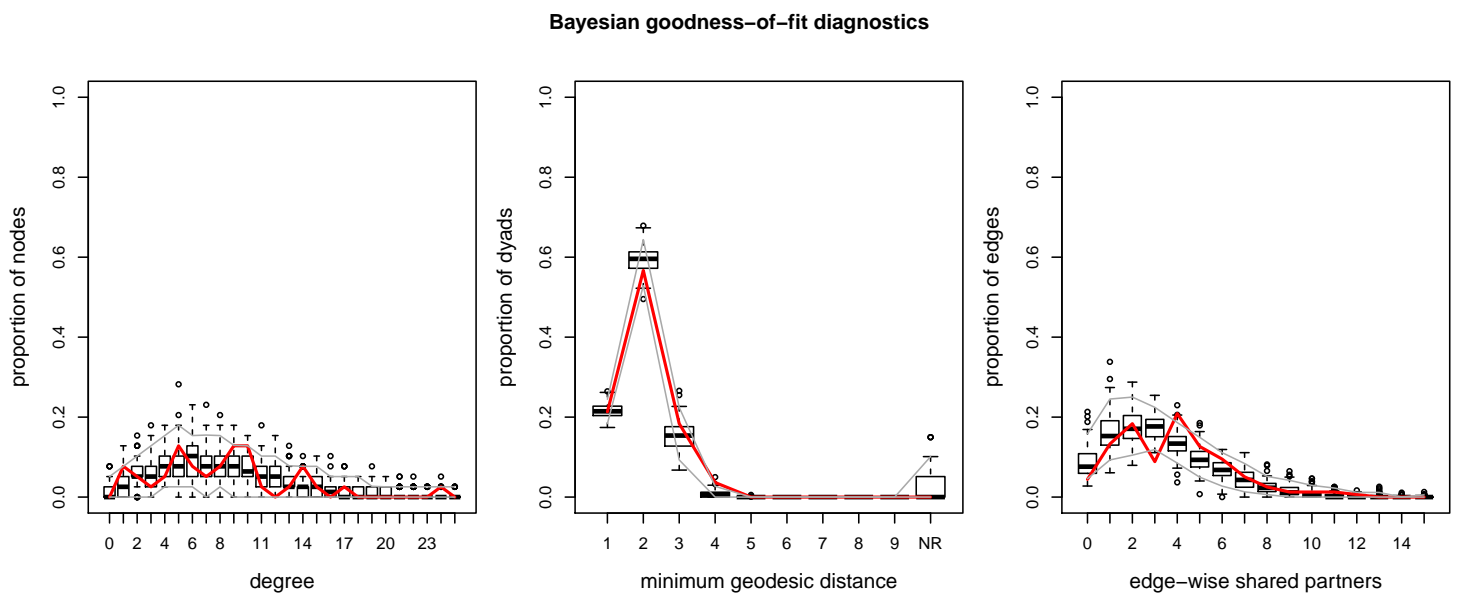

Figure 18 Bayesian goodness-of-fit diagnostics for the model with nodal random effects for the Kapferer data. Bold red line corresponds to original dataset.

Including both effects simultaneously into the model, that means fitting a BERGM with geometrically weighted degree and nodal random effects, would technically be possible, but is not advisable from our experience. The resulting MCMC runs are quite unstable, meaning we get very low acceptance probabilities, and bad mixing behaviour of the chain. Both, geometrically weighted degree and the nodal random effects, are based on the degree of the nodes and we suppose that this leads to some identifiability issues which cause this behaviour.

\subsubsection{European Parliament Members}

The third data example consists of a network of members of the European parliament (MEP) in of the 6th legislative period. The complete network contains more than 900 vertices. We analyse a subset of the 32 members from the Netherlands. The induced subgraph is shown in Figure 19. A link between to MEPs exists if they have at least one committee membership in common. The data were provided by Paul W. Thurner (see Thurner et al., 2013). This data example illustrates our model selection procedure and clearly indicates that not always the more complicated model with more parameters is preferred.

We fitted the same two nested models to the data as in the first part of the karate club example: a standard ERGM with edges and triangles as sufficient statistics, and a model with nodal random effects and the triangle statistic. The number of iterations was also equivalent.

Figures 20 and 21 show the results, which are also summarised in Table 4 . For the mixed model we get a very low acceptance rate for the triangle effect and very high autocorrelations for the triangle effect and the mean parameter $\mu_{\phi}$. The later could possibly be solved by thinning out the chain.

Nevertheless, the focus in this example is on model selection. The computed log Bayes 


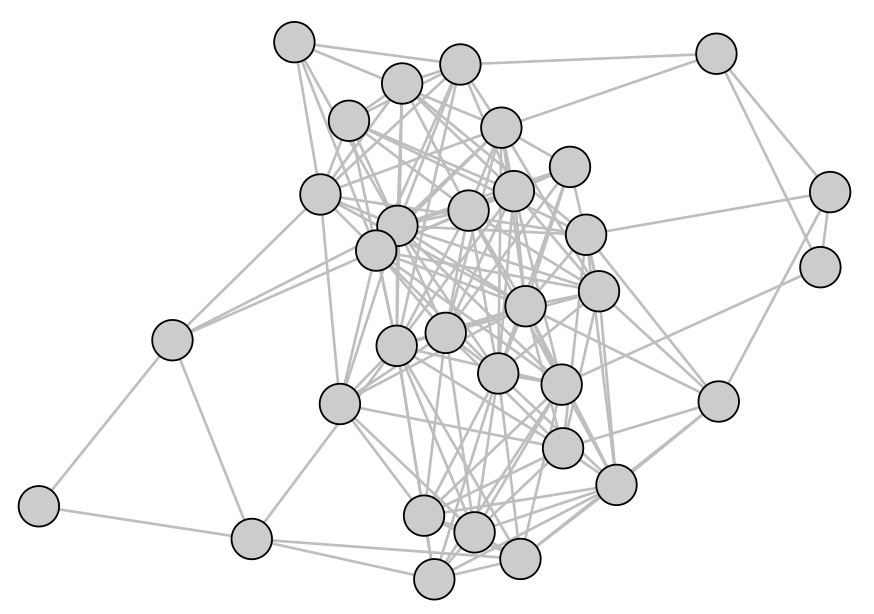

Figure 19 Network of Dutch members of the European parliament during the 6th legislative period. Two members are linked if they have at least one committee membership in common.
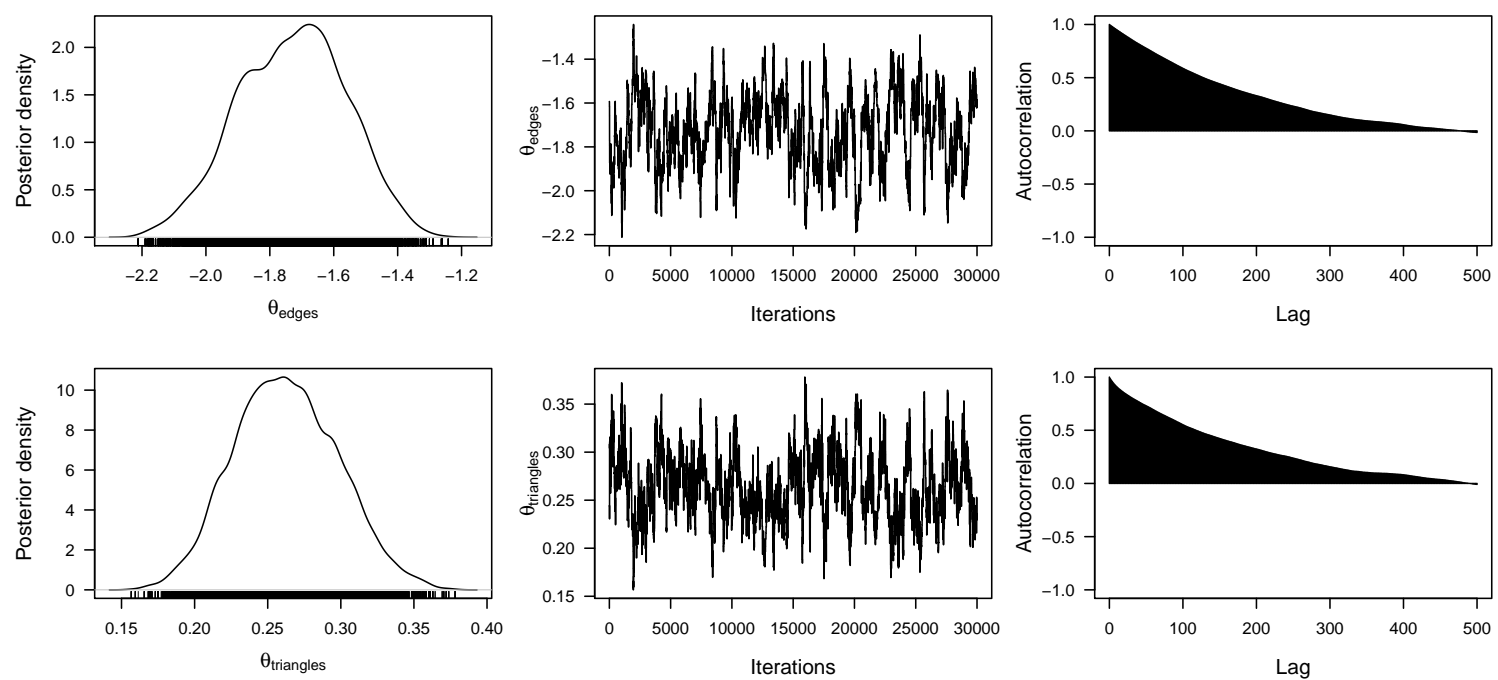

Figure 20 Posterior densities, trace plots, and autocorrelation for the fixed model with edges and triangular effect for the European parliament data.

factor is -13.9 and clearly indicates that the model without nodal random effects is preferable in this situation. Apparently, here we have a network dataset where there is no benefit in including nodal random effects into the model. This corresponds to the rather small estimate for the variance of the nodal random effects $\sigma_{\phi}^{2}$. The resulting Bayes factor shows that it is not the case that the model with more parameters is always selected. This can also be seen from the simulation results in the following subsection. 

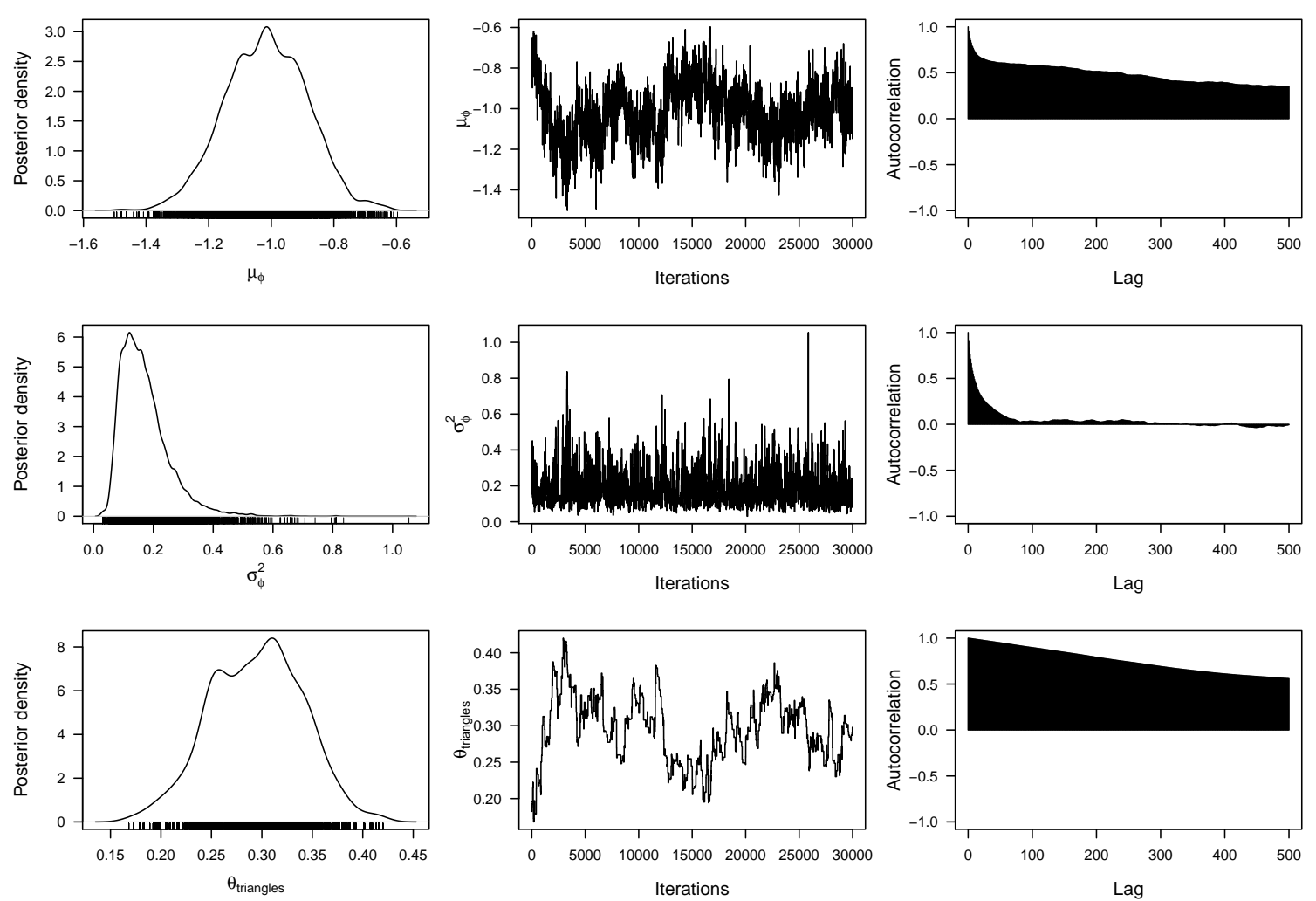

Figure 21 Posterior densities, trace plots, and autocorrelation for the mixed model with nodal random and triangular effects for the European parliament data.

Table 4 Model fitting results for the European parliament data. The fixed model contains edges and triangles, and the mixed model triangles and nodal random effects.

Model type Parameter Post. mean Post. Sd. Acceptance rate Note

\begin{tabular}{llcccc}
\hline \hline \multirow{3}{*}{ fixed } & $\theta_{\text {edges }}$ & -1.73 & 0.17 & & \\
& $\theta_{\text {triangles }}$ & 0.26 & 0.04 & 0.13 & \\
\hline \multirow{4}{*}{ mixed } & $\mu_{\phi}$ & -1.02 & 0.13 & 0.11 & $*$ \\
& $\sigma_{\phi}^{2}$ & 0.15 & 0.08 & 0.15 & $*$ \\
& $\theta_{\text {triangles }}$ & 0.29 & 0.05 & 0.02 &
\end{tabular}

* For $\sigma_{\phi}^{2}$ the posterior mean is calculated based on the logarithmized values and then transformed back to the scale of $\sigma_{\phi}^{2}$ (this leads to the geometric mean) due to the nonsymmetric posterior density in this case. 


\subsection{Simulation}

For the simulation study we used the following components based on two very simple, but different model generating processes, a nodal random effects only situation, i.e. the $p_{2}$ model, and structural effects only situation, i.e. the classical ERGM. For each setting we generated networks with 40 vertices, using again the simulation routines from the ergm package (Hunter et al., 2008). The first model (A) was the one with nodal random effects only, i.e.

$$
\begin{aligned}
\operatorname{logit}\left[\mathbb{P}\left(Y_{i j}=1 \mid Y_{k l},(k, l) \neq(i, j) ; \boldsymbol{\phi}\right)\right] & =\phi_{i}+\phi_{j}, \\
& \text { with } \phi_{i} \sim N\left(\mu_{\phi}, \sigma_{\phi}^{2}\right), \quad \text { for } i=1, \ldots, n .
\end{aligned}
$$

The parameter $\mu_{\phi}$ was constantly set to $\mu_{\phi}=-1$, so that the resulting network graphs tend to be rather sparse. For $\sigma_{\phi}^{2}$ we used values between 0 and 1. Model (B) was the standard ERGM with edges and 2-star statistics, and no nodal random effects, i.e. $\boldsymbol{\theta}=\left(\theta_{\text {edges }}, \theta_{2 \text {-star }}\right)^{t}$ and

$$
\operatorname{logit}\left[\mathbb{P}\left(Y_{i j}=1 \mid Y_{k l},(k, l) \neq(i, j) ; \boldsymbol{\theta}\right)\right]=\theta_{\text {edges }}+\theta_{2 \text {-star }} \cdot\left[\sum_{k \neq j} y_{i k}+\sum_{l \neq i} y_{j l}\right] .
$$

The parameter $\theta_{\text {edges }}$ was constantly set to $\theta_{\text {edges }}=-2$. This is equivalent to model (A) in the sense that $2 \cdot \mu_{\phi}=\theta_{\text {edges }}$, because $\theta_{\text {edges }}$ is a parameter on a per link basis, $\mu_{\phi}$ is

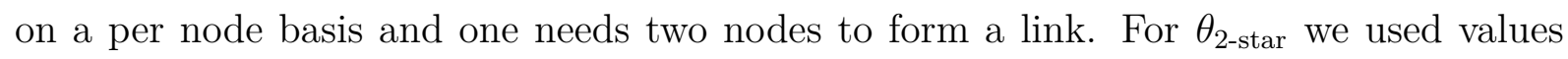
between 0 and 0.05 . This value needs to be small, i.e. close to zero, because otherwise we only generate full or empty graphs if the value is negative, see also Schweinberger (2011). For each of the resulting parameter combinations in model (A) and model (B) we generated 50 networks.

For the chosen settings the resulting 40 node networks seem to be reasonable. We get an average network density between 0.11 and 0.30 for the different settings.

Note that setting $\sigma_{\phi}^{2}=0$ in model $(\mathrm{A})$ and $\theta_{2 \text {-star }}=0$ in model (B) leads to a simple Bernoulli network, which can be seen as a null model.

As a first step, similarly to the karate data example, we fitted two nested models to each of the simulated networks: a standard ERGM with edges and 2-stars as sufficient statistics, and a model with nodal random effects and the 2-star statistic. Again this step was followed by computing a Bayes factor to compare the model with nodal random effects to the one with structural effects only.

Figure 22 shows boxplots of the resulting log Bayes factors for the different settings. For the plot the $\log$ Bayes factors were cut at values of -5 and 5 because some were really small or really large. These cutting values were chosen following Kass and Raftery (1995). More detailed information, especially on the range of the simulation results is given Table 5. For the null model of a pure Bernoulli network the log Bayes factor can point in either one of the directions, the same is more or less true for only small deviations from this null model. The general impression is, that the more extreme the underlying setting becomes the sooner the log Bayes factor points into the correct direction. 
log Bayes factor for mixed against fixed model

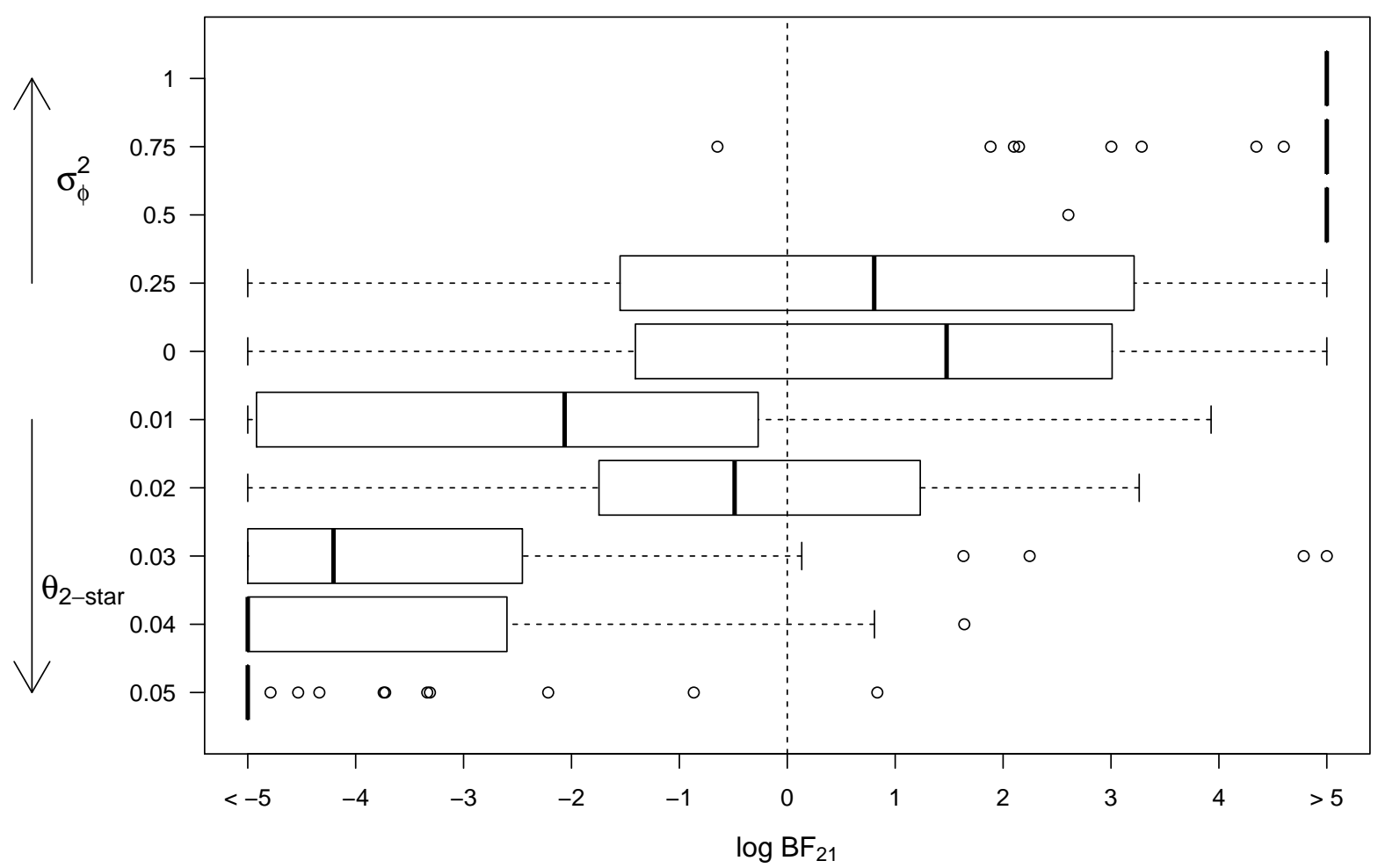

Figure 22 Resulting log Bayes factors for the mixed model against the fixed model (nested models) for different simulation settings. The annotation on the y-axis shows which was the underlying true model, a model with nodal random effects only in the direction of $\sigma_{\phi}^{2}$, and a model with edges and 2-stars in the direction of $\theta_{2-s t a r}$.

Most importantly the results of the simulation show that our model selection works with respect to the size of the competing models. It is not the case that the model with more parameters, which is the model with nodal random effects, is always preferred.

Additionally based on the same simulated networks we compared two non-nested models: the standard ERGM with edges and 2-stars from before, and a model with nodal random effects only. In this case we computed a log Bayes factor for non-nested models as described in Section 3.3. Figure 23 shows the results. Here the distinction seems to be clearer, i.e. the log Bayes factor in most cases clearly indicates the correct direction. In case of the null model the standard ERGM is preffered, i.e. the smaller model which has fewer parameters. 
Table 5 Resulting Bays factors (mixed model against fixed model, nested models) for the simulation from setting (A) a nodal random effects only situation, and setting (B) a classical ERGM with edges and 2-star statistics. Each setting was run 50 times, except for the Bernoulli setting, which had $2 \cdot 50$ runs.

average $\quad \log$ Bayes factor for mixed against fixed model

\begin{tabular}{|c|c|c|c|c|c|c|c|c|}
\hline \multicolumn{2}{|c|}{ Setting } & nw density & $\min$ & $\max$ & $\%<-5$ & $\%<0$ & $\%>0$ & $\%>5$ \\
\hline \multirow{4}{*}{$\begin{array}{c}(\mathrm{A}) \\
\text { random } \\
\text { effects }\end{array}$} & $\sigma_{\phi}^{2}=1$ & 0.23 & 13.03 & 137.64 & 0 & 0 & 100 & 100 \\
\hline & $\sigma_{\phi}^{2}=0.75$ & 0.11 & -0.65 & 498.53 & 0 & 2 & 98 & 84 \\
\hline & $\sigma_{\phi}^{2}=0.5$ & 0.16 & 2.60 & 350.05 & 0 & 0 & 100 & 98 \\
\hline & $\sigma_{\phi}^{2}=0.25$ & 0.15 & -7.73 & 292.34 & 6 & 34 & 66 & 20 \\
\hline \multirow{2}{*}{\multicolumn{2}{|c|}{$\begin{array}{c}\text { Bernoulli network } \\
\sigma_{\phi}^{2}=\theta_{2 \text {-star }}=0\end{array}$}} & & & & & & & \\
\hline & & 0.13 & -7.80 & 10.27 & 4 & 37 & 63 & 4 \\
\hline \multirow{5}{*}{$\begin{array}{c}\text { (B) } \\
\text { fixed } \\
\text { effects }\end{array}$} & $\theta_{2 \text {-star }}=0.01$ & 0.13 & -14.56 & 3.93 & 24 & 76 & 24 & 0 \\
\hline & $\theta_{2 \text {-star }}=0.02$ & 0.14 & -144.23 & 3.26 & 10 & 54 & 46 & 0 \\
\hline & $\theta_{2 \text {-star }}=0.03$ & 0.16 & -25.24 & 51.34 & 44 & 88 & 12 & 2 \\
\hline & $\theta_{2 \text {-star }}=0.04$ & 0.20 & -240.76 & 1.64 & 64 & 94 & 6 & 0 \\
\hline & $\theta_{2 \text {-star }}=0.05$ & 0.30 & -218.07 & 0.83 & 80 & 98 & 2 & 0 \\
\hline
\end{tabular}

Note: For setting $(\mathrm{A})$ we set $\mu_{\phi}=-1$, and for setting $(\mathrm{B}) \theta_{\text {edges }}=-2$, so that $\mu_{\phi}=2 \cdot \theta_{\text {edges }}$. 
$\log$ Bayes factor for random against fixed model

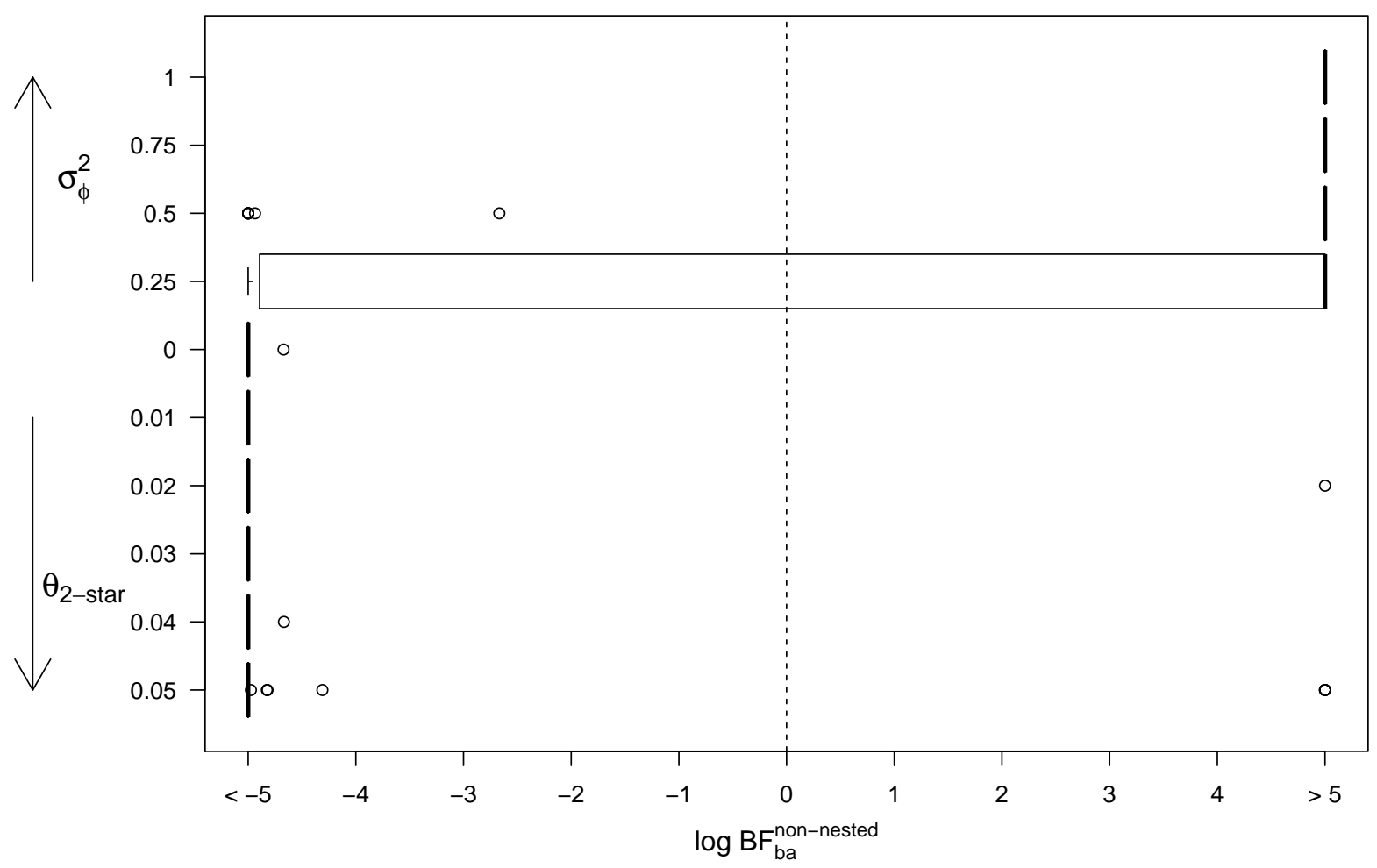

Figure 23 Resulting log Bayes factors for model a with random effects only against model $b$ with fixed effects only (non-nested models) for different simulation settings. The annotation on the $y$-axis shows which was the underlying true model, a model with nodal random effects only in the direction of $\sigma_{\phi}^{2}$, and a model with edges and 2-stars in the direction of $\theta_{2-s t a r}$. 


\section{Discussion and Summary}

Heterogeneity of actors in the network is usually modelled by including (known) nodal or bi-nodal covariates, see, e.g., Robins et al. (2001). Latent, random heterogeneity is considered only exceptionally, for example in Krivitsky et al. (2009), who implicitly assume that the local structure of the network is homogeneous. In particular, this implies that well studied phenomena, such as a small-world networks, Milgram (1967), Watts and Strogatz (1998), where shortest path lengths between two nodes in the network tend to be very small and scale-free networks, where few nodes have unusually high degree, are not appropriately modelled using the standard statistical modelling approaches. This is particularly true for Exponential Random Graph Models.

Here our extension of the Exponential Random Graph Model (ERGM) avoids the assumption of nodal homogeneity. By adding nodal random effects to the model we get a flexible tool to model heterogeneity in the network which is not captured in available (nodal) covariates otherwise. Using the Bayesian framework for ERGMs proposed by Caimo and Friel (2011) allows us to add this random effects extension to the model in an elegant and rather straightforward manner. Estimating Bayes factors enables us to handle the problem of model selection associated with this modelling task. The resulting estimates for the three data examples seem to be reasonable.

Furthermore, the small simulation study in the previous section suggests that in general the Bayes factor approach seems to work and even though a mixed model with nodal random effects has more parameters than its fixed equivalent it is not systematically preferred in the model selection.

The model can at least conceptually be extended to allow for node specific network effects. In this case one replaces the right hand side in model (5) through $\boldsymbol{\theta}^{t} s_{i j}(\boldsymbol{y})+\tilde{s}_{i j}(\boldsymbol{y})\left(\phi_{i}+\phi_{j}\right)$, where $\tilde{s}_{i j}(\boldsymbol{y})$ is a subvector of $s_{i j}(\boldsymbol{y})$. In this case one may, for instance, model that the 2-star effect is heterogeneous amongst the nodes in the network. However, even though conceptually this is straight-forward, the computation in the Bayesian estimation is even more challenging.

We should note that the approach which we have introduced is computationally intensive. A promising avenue of research to address this issue is to explore approximations of the likelihood function using composite likelihoods, of which the pseudolikelihood approximation Frank and Strauss (1986) is an antecedent. We refer the reader to Varin et al. (2011) for a recent review of composite likelihoods. We are currently engaged in work in this direction.

Secondly, as parallel computing is becoming more accessible this will help to shorten the time for computation. This also applies to parallel computing in the Gibbs step of the algorithm. 


\section{A Laplace approximation}

The likelihood in the mixed effects model marginalized over the random effects $\phi$ is

$$
\begin{aligned}
f\left(\boldsymbol{y} \mid \boldsymbol{\theta}, \mu_{\phi}, \sigma_{\phi}^{2}\right)= & \int \frac{\exp \left\{\boldsymbol{\theta}^{t} s(\boldsymbol{y})+\boldsymbol{\phi}^{t} t(\boldsymbol{y})\right\}}{\kappa(\boldsymbol{\theta}, \boldsymbol{\phi})} \cdot p\left(\boldsymbol{\phi} \mid \mu_{\phi}, \sigma_{\phi}^{2}\right) \mathrm{d} \boldsymbol{\phi} \\
= & \int \frac{\exp \left\{\boldsymbol{\theta}^{t} s(\boldsymbol{y})+\boldsymbol{\phi}^{t} t(\boldsymbol{y})\right\}}{\kappa(\boldsymbol{\theta}, \boldsymbol{\phi})} \cdot \frac{1}{(2 \pi)^{\frac{n}{2}}\left|\sigma_{\phi}^{2} I_{n}\right|^{\frac{1}{2}}} \cdot \\
& \cdot \exp \left\{-\frac{1}{2 \sigma_{\phi}^{2}}\left(\boldsymbol{\phi}-\mu_{\phi} \mathbb{1}_{n}\right)^{t}\left(\boldsymbol{\phi}-\mu_{\phi} \mathbb{1}_{n}\right)\right\} \mathrm{d} \boldsymbol{\phi} \\
= & \frac{\exp \left\{\boldsymbol{\theta}^{t} s(\boldsymbol{y})\right\}}{\left(2 \pi \sigma_{\phi}^{2}\right)^{\frac{n}{2}}} \cdot \\
& \cdot \int \exp \left\{\boldsymbol{\phi}^{t} t(\boldsymbol{y})-\frac{1}{2 \sigma_{\phi}^{2}}\left(\boldsymbol{\phi}-\mu_{\phi} \mathbb{1}_{n}\right)^{t}\left(\boldsymbol{\phi}-\mu_{\phi} \mathbb{1}_{n}\right)-\log (\kappa(\boldsymbol{\theta}, \boldsymbol{\phi}))\right\} \mathrm{d} \boldsymbol{\phi} .
\end{aligned}
$$

The integral in equation (19) is approximated around the point $\widehat{\phi}$ using a Laplace type approximation (Severini, 2000, section 2.11)

$$
\int \exp \{-h(\phi)\} \mathrm{d} \phi \approx \exp \{-h(\widehat{\phi})\}(2 \pi)^{\frac{n}{2}}|\Sigma|^{-\frac{1}{2}},
$$

where

$$
h(\boldsymbol{\phi})=-\boldsymbol{\phi}^{t} t(\boldsymbol{y})+\frac{1}{2 \sigma_{\phi}^{2}}\left(\boldsymbol{\phi}-\mu_{\phi} \mathbb{1}_{n}\right)^{t}\left(\boldsymbol{\phi}-\mu_{\phi} \mathbb{1}_{n}\right)+\log (\kappa(\boldsymbol{\theta}, \boldsymbol{\phi}))
$$

and

$$
\begin{aligned}
\Sigma & =\frac{\partial^{2} h(\widehat{\boldsymbol{\phi}})}{\partial \widehat{\boldsymbol{\phi}} \partial \widehat{\boldsymbol{\phi}}^{t}} \\
& =\frac{1}{\sigma_{\phi}^{2}} I_{n}+\frac{\partial^{2}}{\partial \widehat{\boldsymbol{\phi}} \partial \widehat{\boldsymbol{\phi}}^{t}} \log (\kappa(\boldsymbol{\theta}, \widehat{\boldsymbol{\phi}})) \\
& =\frac{1}{\sigma_{\phi}^{2}} I_{n}+\operatorname{Cov}\left(t(\boldsymbol{Y}), t(\boldsymbol{Y})^{t} \mid \widehat{\boldsymbol{\phi}}, \boldsymbol{\theta}\right) .
\end{aligned}
$$

The matrix $\operatorname{Cov}\left(t(\boldsymbol{Y}), t(\boldsymbol{Y})^{t} \mid \widehat{\boldsymbol{\phi}}, \boldsymbol{\theta}\right)$ denotes the covariance matrix of the vector of degree statistics $t(\boldsymbol{Y})$ and can be estimated via simulated networks using the parameters $\widehat{\phi}$ and $\boldsymbol{\theta}$. These networks are drawn in the same way as the auxiliary networks needed for the exchange algorithm described in Section 2.

We assume that the posterior mode is close to the maximum likelihood estimator. The two are identical if the prior distributions are non-informative. This is not the case here, but we are assuming flat prior distributions and therefore the two should be reasonably close to each other. For reasons of simplicity, we use the posterior mean as value for $\widehat{\phi}$. Combining equation (19) with equation (20) yields

$$
f\left(\boldsymbol{y} \mid \boldsymbol{\theta}, \mu_{\phi}, \sigma_{\phi}^{2}\right) \approx \frac{\exp \left\{\boldsymbol{\theta}^{t} s(\boldsymbol{y})\right\}}{\kappa(\boldsymbol{\theta}, \widehat{\boldsymbol{\phi}})} \widehat{f}_{\text {Laplace }}\left(\boldsymbol{y} \mid \widehat{\boldsymbol{\phi}}, \mu_{\phi}, \sigma_{\phi}^{2}\right),
$$


with

$$
\widehat{f}_{\text {Laplace }}\left(\boldsymbol{y} \mid \widehat{\boldsymbol{\phi}}, \mu_{\phi}, \sigma_{\phi}^{2}\right)=\sigma_{\phi}^{-n} \exp \left\{\widehat{\boldsymbol{\phi}}^{t} t(\boldsymbol{y})-\frac{1}{2 \sigma_{\phi}^{2}}\left(\widehat{\boldsymbol{\phi}}-\mu_{\phi} \mathbb{1}_{n}\right)^{t}\left(\widehat{\boldsymbol{\phi}}-\mu_{\phi} \mathbb{1}_{n}\right)\right\}|\Sigma|^{-\frac{1}{2}}
$$




\section{References}

Caimo, A. and Friel, N. (2011). Bayesian inference for exponential random graph models. Social Networks, 33(1):41-55.

Caimo, A. and Friel, N. (2013). Bayesian model selection for exponential random graph models. Social Networks, 35(1):11-24.

Caimo, A. and Friel, N. (2014). Bergm: Bayesian exponential random graphs in R. Journal of Statistical Software, 61(2):1-25.

Chatterjee, S. and Diaconis, P. (2013). Estimating and understanding exponential random graph models. The Annals of Statistics, 41(5):2428-2461.

Everitt, R. G. (2012). Bayesian parameter estimation for latent markov random fields and social networks. Journal of Computational and Graphical Statistics, 21(4):940-960.

Fellows, I. and Handcock, M. S. (2012). Exponential-family random network models. arXiv preprint arXiv:1208.0121.

Fienberg, S. E. (2012). A Brief History of Statistical Models for Network Analysis and Open Challenges. Journal of Computational and Graphical Statistics, 21(4):825-839.

Frank, O. and Strauss, D. (1986). Markov graphs. Journal of the American Statistical Association, 81(395):832-842.

Gelman, A. and Meng, X.-L. (1998). Simulating normalizing contants: from importance sampling to bridge sampling to path sampling. Statistical Science, 13(2):163-185.

Geyer, C. J. and Thompson, E. A. (1992). Constrained Monte Carlo maximum likelihood for dependent data. Journal of the Royal Statistical Society. Series B (Methodological), 54(3):657-699.

Gill, P. S. and Swartz, T. B. (2004). Bayesian analysis of directed graphs data with application to social networks. Journal of the Royal Statistical Society: Series C (Applied Statistics), 53(2):249-260.

Goldenberg, A., Zheng, A. X., Fienberg, S. E., and Airoldi, E. M. (2010). A survey of statistical network models. Foundations and Trends in Machine Learning, 2(2):129-233.

Holland, P. W. and Leinhardt, S. (1981). An exponential family of probability distributions for directed graphs. Journal of the American Statistical Association, 76(373):33-50.

Hunter, D. R. (2007). Curved exponential family models for social networks. Social Networks, 29(2):216-230.

Hunter, D. R. and Handcock, M. S. (2006). Inference in curved exponential family models for networks. Journal of Computational and Graphical Statistics, 15(3):565-583. 
Hunter, D. R., Handcock, M. S., Butts, C. T., Goodreau, S. M., and Morris, M. (2008). ergm: A package to fit, simulate and diagnose exponential-family models for networks. Journal of Statistical Software, 24(3):1-29.

Hunter, D. R., Krivitsky, P. N., and Schweinberger, M. (2012). Computational statistical methods for social network analysis. Journal of Computational and Graphical Statistics, $21(4): 856-882$.

Kapferer, B. (1972). Strategy and Transaction in an African Factory: African Workers and Indian Management in a Zambian Town. Manchester University Press.

Kass, R. E. and Raftery, A. E. (1995). Bayes Factors. Journal of the American Statistical Association, 90(430):773-795.

Kolaczyk, E. D. (2009). Statistical Anaysis of Network Models. Springer, New York.

Krivitsky, P. N., Handcock, M. S., Raftery, A. E., and Hoff, P. D. (2009). Representing degree distributions, clustering, and homophily in social networks with latent cluster random effects models. Social Networks, 31(3):204-213.

Lusher, D., Koskinen, J., and Robins, G. (2013). Exponential Random Graph Models for Social Networks. Cambridge University Press, Cambridge.

Milgram, S. (1967). The small world problem. Psychology Today, 2(1):60-67.

Murray, I., Ghahramani, Z., and MacKay, D. (2006). MCMC for doubly-intractable distributions. In Proceedings of the 22nd Annual Conference on Uncertainty in Artificial Intelligence (UAI-06), Arlington, Virginia. AUAI Press.

R Core Team (2015). R: A Language and Environment for Statistical Computing. $\mathrm{R}$ Foundation for Statistical Computing, Vienna, Austria.

Robins, G. and Lusher, D. (2013). Illustrations: Simulation, estimation, and goodness of fit. In Lusher et al. (2013), pages 167-185.

Robins, G. L., Elliot, P., and Pattison, P. (2001). Network models for social selection processes. Social Networks, 23(1):1-30.

Robins, G. L., Pattison, P., Kalish, Y., and Lusher, D. (2007a). An introduction to exponential random graph $\left(p^{*}\right)$ models for social networks. Social Networks, 29(2):173191.

Robins, G. L., Snijders, T. A. B., Wang, P., Handcock, M. S., and Pattison, P. (2007b). Recent developments in exponential random graph $\left(p^{*}\right)$ models for social networks. Social Networks, 29(2):192-215.

Salter-Townshend, M., White, A., Gollini, I., and Murphy, T. B. (2012). Review of statistical network analysis: models, algorithms, and software. Statistical Analysis and Data Mining, 5(4):243-264. 
Schweinberger, M. (2011). Instability, Sensitivity, and Degeneracy of Discrete Exponential Families. Journal of the American Statistical Association, 106(496):1361--1370.

Severini, T. (2000). Likelihood Methods in Statistics. Oxford science publications. Oxford University Press.

Snijders, T. A. B., Pattison, P. E., Robins, G. L., and Handcock, M. S. (2006). New specifications for exponential random graph models. Sociological Methodology, 36(1):99153.

Spiegelhalter, D. J., Best, N. G., Carlin, B. P., and van der Linde, A. (2002). Bayesian measures of model complexity and fit. Journal of the Royal Statistical Society: Series $B$ (Statistical Methodology), 64(4):583-639.

Strauss, D. and Ikeda, M. (1990). Pseudolikelihood estimation for social networks. Journal of the American Statistical Association, 85(409):204-212.

Thurner, P. W., Kiel, M., and Schneider, M. (2013). Committee networks in the european parliament: Structure and impact on allocation of reports. Ms. LMU Munich.

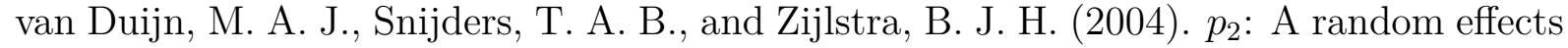
model with covariates for directed graphs. Statistica Neerlandica, 58(2):234-254.

Varin, C., Reid, N., and Firth, D. (2011). An overview of composite likelihood methods. Statistica Sinica, 21(1):5-42.

Watts, D. J. and Strogatz, S. H. (1998). Collective dynamics of 'small-world' networks. Nature, 393(6684):440-442.

Zachary, W. W. (1977). An information flow model for conflict and fission in small groups. Journal of Anthropological Research, 33(4):452-473.

Zijlstra, B. J. H., Duijn, M. A. J., and Snijders, T. A. B. (2006). The multilevel $p_{2}$ model: A random effects model for the analysis of multiple social networks. Methodology: European Journal of Research Methods for the Behavioral and Social Sciences, 2(1):4247. 


\section{Acknowledgements}

We gratefully acknowledge the data provision by Paul W. Thurner.

The Insight Centre for Data Analytics is supported by Science Foundation Ireland under Grant Number SFI/12/RC/2289. Nial Friel's research was also supported by an Science Foundation Ireland grant: 12/IP/1424.

Alberto Caimo's research was supported by the Swiss National Science Foundation (SNSF), under grant: CR12I1-156229. 